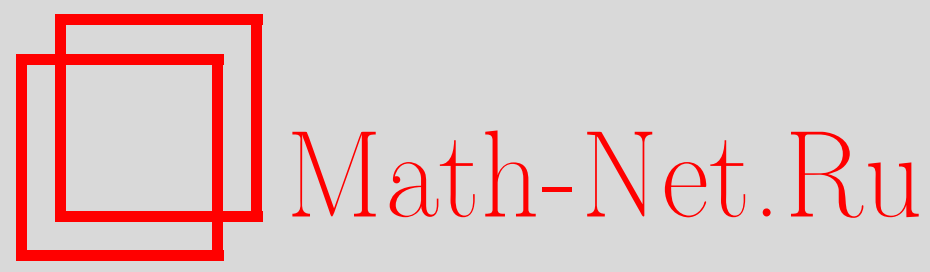

Б. С. Кругликов, Тензоры Ниенхейса и препятствия к построению псевдоголоморфных отображений, Матем. заметки, 1998, том 63, выпуск 4, 541-561

DOI: https://doi.org/10.4213/mzm1315

Использование Общероссийского математического портала Math-Net.Ru подразумевает, что вы прочитали и согласны с пользовательским соглашением http://www . mathnet.ru/rus/agreement

Параметры загрузки:

IP: 54.92 .164 .108

26 апреля 2023 г., $17: 36: 15$

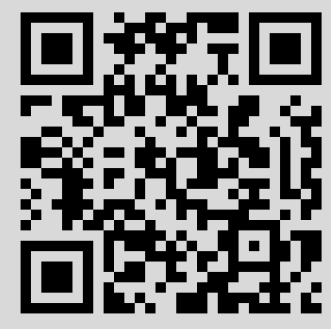




\section{ТЕНЗОРЫ НИЕНХЕЙСА И ПРЕПЯТСТВИЯ К ПОСТРОЕНИЮ ПСЕВДОГОЛОМОРФНЫХ ОТОБРАЖЕНИЙ}

\section{Б. С. Кругликов}

В работе предложено несколько подходов к классификации почти комплексных структур и построению локальных или формальных псевдоголоморфных отображений из одного почти комплексного многообразия в другое. Соответствуюшие критерии даны в терминах тензоров Ниенхейса и их обобщений.

Библиографой: 14 названий.

\section{Введение}

Пусть $\left(L^{2 l}, j_{L}\right)$ и $\left(M^{2 m}, j_{M}\right)$ - почти комплексные многообразия, т.е. многообразия, снабженные автоморфизмами $j_{L}$ и $j_{M}$ касательного расслоения, удовлетворяющими соотношениям $j_{L}^{2}=-\mathbf{1}_{L}: T_{*} L \rightarrow T_{*} L$ и $j_{M}^{2}=-\mathbf{1}_{M}$. Псевдоголоморфным отображением (РН-отображсением) назьвается отображение $u: L \rightarrow M$ такое, что его дифференциал $\left.u_{*}\right|_{x}=d u_{x}: T_{x} L \rightarrow T_{u(x)} M$ коммутирует с почти комплексной структурой: $j_{M} \circ u_{*}=u_{*} \circ j_{L}$.

Если $M=\mathbb{C}$, то (нетривиальное) псевдоголоморфное отображение $u: L \rightarrow M$ называется комплексной координатой, и в общем случае такой координаты не существует (более того, если на многообразии $L^{2 l}$ существует $l$ независимых локальных комплексных координат, то оно является комплексным). Для $l=1$, т.е. когда $\left(L, j_{L}\right)$ - риманова поверхность, РН-отображения существуют [1] и, более того, семейство таких отображений обладает структурой многообразия (при некоторых условиях, см. [2], [3]). Если же $l>1$, то для почти комплексной структуры $j_{L}$ общего вида такого отображения не существует даже локально. Препятствием является тензор Ниенхейса. Хорошо известно [1], [4], что если тензор Ниенхейса тождественно равен 0, то почти комплексное многообразие является комплексным, и для двух таких многообразий $L$ и $M$ сушествует локальное голоморфное отображение $u:(L, x) \rightarrow(M, y)($ см. также [5]). В этой работе мы рассматриваем условия существования формального и локального псевдоголоморфных отображений для общих почти комплексных структур.

В 11 мы доказываем необходимое и достаточное условие для продолжения псевдоголоморфного на уровне 1-струй отображения одного почти комплексного многообразия в другое до псевдоголоморфного отображения на уровне 2-струй. Другими словами, мы описываем первое продолжение соответствующего уравнения Коши-Римана на пространстве 1-струй. В данном случае уравнение Коши-Римана - это условие коммутирования дифференциала $\Phi=d u$ искомого отображения $u$ в точке с почти комплексными структурами: $j_{M} \circ \Phi=\Phi \circ j_{L}$. Мы вводим пространство $k$-струй псевдоголоморфных отображений, которое в общем случае не имеет структуры многообразия, a 
для формально интегрируемых “пополненных" уравнений Коши-Римана имеет. Здесь под "пополнением" подразумевается добавление определяющих соотношений, т.е. сужение в смысле пространства струй [6]. Уравнение Коши-Римана для отображений одного почти комплексного многообразия в другое является формально интегрируемым только в интегрируемом случае, т.е. когда многообразия комплексны. В общем случае это уравнение надо "пополнять". Первьм членом “пополнения" является условие коммутирования дифференциала искомого отображения в точке с тензорами Ниенхейса: $N_{j_{M}} \circ \wedge^{2} \Phi=\Phi \circ N_{j_{L}}$

В $\S 2$ мы рассматриваем вопрос формальной интегрируемости, вводим новый инвариант - тензор Ниенхейса второго порядка $N_{j}^{(2)}$ - и выписьваем следуюший член “пополнения": $N_{j_{M}}^{(2)} \circ \wedge^{2}\left(\wedge^{2} \Phi\right)=\Phi \circ N_{j_{L}}^{(2)}$

В $\S 3$ мы приводим критерий разрешимости уравнения Коши-Римана на уровне высших струй. Как следствие, мы получаем формальную часть известной теоремы Ньюландера-Ниренберга об интегрируемости почти комплексной структуры. Мы также используем этот критерий для нахождения формальных нормальных форм почти комплексных структур.

В $\S 4$ мы вводим пространство линейных тензоров Ниенхейса и рассматриваем вопрос восстановления линейной комплексной структуры по тензору Ниенхейса.

В 55 мы определяем понятие тензоров Ниенхейса общего вида. Мы назьваем почти комплексную структуру структурой общего вида, если соответствующий тензор Ниенхейса имеет общий вид. Для многообразий, снабженных такими структурами, необходимое и достаточное условие существования псевдоголоморфного отображения может быть сформулировано только в терминах тензоров Ниенхейса, что и составляет утверждение теоремы 6.

В $\S 6$ мы приводим классификацию почти комплексных структур общего вида на четырехмерньх многообразиях в терминах распределений (п. 6.1), а также вводим понятие лиевой почти комплексной структуры и приводим классификацию таких структур общего вида (п. 6.2).

Параграфы 4-6 не используют результатов и методов параграфов 1-3 и могут быть прочитаны независимо.

\section{§ 1. Тензор Ниенхейса как первое препятствие к построению псевдоголоморфного отображения}

Для отображения $u:(L, x) \rightarrow(M, y)$ обозначим через $u_{*}^{\wedge k}: \wedge^{k} T_{x} L \rightarrow \wedge^{k} T_{y} M$ индуцированное отображение.

Обозначим через $\mu=\mu_{x}$ идеал в кольце гладких функций $C^{\infty}(L)$, обращающихся в точке $x$ в 0 . Степень этого идеала $\mu^{k}$ является идеалом в кольце функций, обращаюшихся в 0 в точке $x$ вместе со своими производными порядка меньше $k: \mu^{k}=\{f \mid$ $\left.\partial^{\sigma} f(x)=0,|\sigma|<k\right\}$. Будем обозначать через $\mu^{k}=\mu^{k} T(p, q)$ подмодуль в модуле тензоров типа $(p, q)$, имеющих в точке $x$ нуль порядка $k$. Обозначим символом $\mathscr{O}_{L}(x)$ росток окрестностей точки $x \in L$. Положим $\mu_{x, y}^{k}=\left\{\Phi: T \mathscr{O}_{L}(x) \rightarrow T \mathscr{O}_{M}(y) \mid \operatorname{Im} \Phi \subset \mu_{y}^{k}\right\}$.

Формальным псевдоголоморфным отображсением назьвается отображение $u$ : $\mathscr{O}_{L}(x) \rightarrow \mathscr{O}_{M}(y)$ такое, что $j_{M} \circ u_{*}-u_{*} \circ j_{L} \in \mu^{\infty}$. Это отображение назьвается нетривиальным, если $u_{*} \notin \mu^{\infty}$. Более того, в основном мы будем рассматривать невырожденные отображения: $u_{*} \notin \mu$ или $d u_{x} \neq 0$.

Рассмотрим тензоры Ниенхейса $N_{j_{L}}$ и $N_{j_{M}}$ на многообразиях $L$ и $M$ :

$$
N_{j}(\xi, \eta)=[j \xi, j \eta]-j[j \xi, \eta]-j[\xi, j \eta]-[\xi, \eta] .
$$


Непосредственно из определения следует, что если $u: L \rightarrow M-$ псевдоголоморфное отображение, то $N_{j_{M}} \circ u_{*}^{\wedge 2}=u_{*} \circ N_{j_{L}}$. Более того, это равенство вьполнено в точке $x$, если $u$ сохраняет почти комплексную структуру с точностью до второго порядка: $j_{M} \circ u_{*}-u_{*} \circ j_{L} \in \mu_{x, y}^{2}$.

Обозначим $J_{\mathrm{PH}}^{0}(L, M)=J^{0}(L, M)=L \times M$ (используем обозначения из теории пространств струй, см. [7], [8]). Обозначим через $J_{\mathrm{PH}}^{1}(L, M)$ подрасслоение расслоения струй $J^{1}(L, M) \rightarrow J^{0}(L, M)$ со слоем

$$
\left(J_{\mathrm{PH}}^{1}\right)_{x, y}=\left\{\Phi: T_{x} L \rightarrow T_{y} M \mid j_{M} \circ \Phi=\Phi \circ j_{L}\right\} .
$$

Другими словами, многообразие $J_{\mathrm{PH}}^{1}$ является расслоением над $L \times M$ со слоем $\left(J_{\mathrm{PH}}^{1}\right)_{x, y}$. Обозначим также

$$
J_{\mathrm{PH}}^{k} \supset\left(J_{\mathrm{PH}}^{k}\right)_{x, y}=\frac{\left\{u_{*}: T \mathscr{O}_{L}(x) \rightarrow T \mathscr{O}_{M}(y) \mid j_{L} \circ u_{*}-u_{*} \circ j_{M} \in \mu_{x, y}^{k}\right\}}{\left\{u_{*}: T \mathscr{O}_{L}(x) \rightarrow T \mathscr{O}_{M}(y) \mid u_{*} \in \mu_{x, y}^{k}\right\}} .
$$

В отличие от $J_{\mathrm{PH}}^{1}$ множества $J_{\mathrm{PH}}^{k}, k \geqslant 2$, вообще говоря, не имеют структуры многообразия. Это связано с тем, что для отображения $\pi_{\mathrm{PH}}^{r, s}$ ограничения проекции $\pi^{r, s}$ : $J^{r}(L, M) \rightarrow J^{s}(L, M), r \geqslant s$, прообраз точки может быть пуст.

Для получения критерия сушествования формального РН-отображения применим метод Картана продолжений-проекций [6]. Обозначим через $\mathscr{E} k$ образ проекции $\operatorname{Im} \pi_{\mathrm{PH}}^{k, 1} \subset J_{\mathrm{PH}}^{1} \subset J^{1}(L, M)$. Можно трактовать эти подмножества в пространстве 1-струй как дифференциальные уравнения первого порядка. Проблема построения формального псевдоголоморфного отображения сводится к сужению уравнения нахождению проективного предела

$$
\mathscr{E}^{\infty} \subset \cdots \subset \mathscr{E}^{2} \subset \mathscr{E}^{1} \subset J^{1}(L, M) .
$$

Уравнение Коши-Римана $\mathscr{E}^{1}=J_{\mathrm{PH}}^{1}$ расслоено над $J^{0}=L \times M$ со слоем

$$
\mathscr{E}_{x, y}^{1}=\left(\pi_{\mathrm{PH}}^{1,0}\right)^{-1}(x, y)=\left\{\Phi: T_{x} L \rightarrow T_{y} M \mid j_{M} \circ \Phi=\Phi \circ j_{L}\right\} .
$$

ТЕОРема 1. Ограничение проекиии $\pi_{\mathrm{PH}}^{1,0}$ на уравнение $\mathscr{E}^{2} \subset J_{\mathrm{PH}}^{1}$ имеет прообра$3 b l$

$$
\mathscr{E}_{x, y}^{2}=\left\{\Phi: T_{x} L \rightarrow T_{y} M \mid j_{M} \circ \Phi=\Phi \circ j_{L}, N_{j_{M}} \circ \Phi^{\wedge 2}=\Phi \circ N_{j_{L}}\right\}
$$

Доказательства этой и остальных теорем потребуют вычислений в пространствах струй. С этой целью введем следующие конструкции.

ОПРЕДЕЛЕНИЕ 1. Для любого векторного поля $\xi$ на $X$ зададим отображения $d^{p} \xi=$ $d_{\nabla}^{p} \xi: \bigotimes_{1}^{p} T_{x} X \rightarrow T_{x} X, x \in X$, формулой

$$
\begin{gathered}
d \xi(\eta)=\nabla_{\eta} \xi, \quad d^{2} \xi\left(\eta_{1}, \eta_{2}\right)=\nabla_{\eta_{2}} \nabla_{\eta_{1}}(\xi)-\nabla_{\nabla_{\eta_{2}} \eta_{1}}(\xi), \\
d^{p+1} \xi\left(\eta_{1}, \ldots, \eta_{p+1}\right)=d\left(d^{p} \xi\left(\eta_{1}, \ldots, \eta_{p}\right)\right)\left(\eta_{p+1}\right)-\sum_{i=1}^{p} d^{p} \xi\left(\eta_{1}, \ldots, d \eta_{i}\left(\eta_{p+1}\right), \ldots, \eta_{p}\right),
\end{gathered}
$$

$p \geqslant 2$, где $\nabla$ - некоторая симметричная связность. Если кривизна связности $\nabla$ равна 0 , то дифференциал $d_{\nabla}^{p} \xi$ симметричен, $d_{\nabla}^{p} \xi: \mathbb{S}^{p} T_{x} X \rightarrow T_{x} X$, и в локальных евклидовых координатах имеет вид

$$
d^{p} \xi\left(\eta_{1}, \ldots, \eta_{p}\right)^{i}=\frac{\partial^{p} \xi^{i}}{\partial x^{i_{1}} \cdots \partial x^{i_{p}}} \eta_{1}^{i_{1}} \cdots \eta_{p}^{i_{p}} .
$$

Легко видеть, что для $d \xi=d^{1} \xi$ выполнено $L_{\xi} \eta=[\xi, \eta]=d \eta(\xi)-d \xi(\eta)$. 
ОПРЕДЕЛЕНИЕ 2. Для произвольного морфизма расслоений $A=\left(A_{x}\right)_{x \in X}, A_{x}$ : $\wedge^{q} T_{x} X \rightarrow T_{x} X$, определим отображения $d^{p} A, d^{p} A_{x}: \wedge^{q} T_{x} X \otimes\left(\otimes_{1}^{p} T_{x} X\right) \rightarrow T_{x} X$, индуктивно: $d^{0} A:=A$,

$$
\begin{aligned}
d^{p+1} A\left(\xi_{1}, \ldots, \xi_{q+p+1}\right)= & d\left(d^{p} A\left(\xi_{1}, \ldots, \xi_{q+p}\right)\right)\left(\xi_{q+p+1}\right) \\
& -\sum_{i=1}^{q+p} d^{p} A\left(\xi_{1}, \ldots, d \xi_{i}\left(\xi_{q+p+1}\right), \ldots, \xi_{q+p}\right) .
\end{aligned}
$$

Если кривизна связности $\nabla$ равна 0 , то связность является локально тривиальной и дифференциал $d^{p} A: \wedge^{q} T_{x} X \otimes \mathbb{S}^{p} T_{x} X \rightarrow T_{x} X$ в локальных евклидовых координатах имеет вид

$$
\left[d^{p} A\left(\xi_{1}, \ldots, \xi_{q+p}\right)\right]^{i}=\sum_{i_{1}<\cdots<i_{q}} \frac{\partial^{p} A_{i_{1} \ldots i_{q}}^{i_{1}}}{\partial x^{i_{q+1}} \cdots \partial x^{i_{q+p}}} \xi_{1}^{i_{1}} \cdots \xi_{q}^{i_{q}} \cdots \xi_{q+p}^{i_{q+p}} .
$$

Итак, евклидова связность $\nabla$ определяет отображение $d_{\nabla}^{p}: \Omega^{q} \otimes \mathscr{D} \rightarrow \Omega^{q} \otimes \mathbb{S}^{p} \Omega^{1} \otimes \mathscr{D}$, где $\mathscr{D}$ - модуль векторных полей, а $\Omega^{q}$ - модуль внешних форм. Заметим, что такое отображение может быть построено по произвольной связности $\nabla$ (см. [9]), но ввиду локальности дальнейших рассуждений будем считать связность $\nabla$ тривиальной (евклидовой). Будем писать $d^{p}$ вместо $d_{\nabla}^{p}$.

ДоКАЗАТЕЛЬСТВо ТЕОРЕМЫ 1. 1-РН-струя $\Phi$ поднимается до 2-РН-струи тогда и только тогда, когда выполнены уравнения

$$
\begin{gathered}
j_{M} \circ \Phi(\xi)=\Phi \circ j_{L}(\xi), \quad \Phi^{(2)}(\xi, \eta)=\Phi^{(2)}(\eta, \xi), \\
d j_{M}(\Phi \xi, \Phi \eta)+j_{M} \circ \Phi^{(2)}(\xi, \eta)=\Phi^{(2)}\left(j_{L} \xi, \eta\right)+\Phi \circ d j_{L}(\xi, \eta),
\end{gathered}
$$

где $\xi, \eta \in T_{x} L$, а $\Phi^{(2)}: \mathbb{S}^{2} T_{x} L \rightarrow T_{y} M$ есть 2-символ искомого РН-отображения, которьй в локальных координатах имеет вид

$$
\Phi^{(2)}(\xi, \eta)^{i}=\frac{\partial^{2} u^{i}}{\partial x^{r} \partial x^{s}} \xi^{r} \eta^{s}
$$

Рассмотрим тензор

$$
P \in T_{x}^{*} L \otimes T_{x}^{*} L \otimes T_{y} M, \quad P(\xi, \eta)=j_{M} \circ \Phi^{(2)}(\xi, \eta)-\Phi^{(2)}\left(j_{L} \xi, \eta\right) .
$$

Из (1) имеем

$$
P(\xi, \eta)=\Phi \circ d j_{L}(\xi, \eta)-d j_{M}(\Phi \xi, \Phi \eta) .
$$

Лемма 1. Тензор $P$, определенны й по симметричному тензору $\Phi^{(2)}$ формулой (2), удовлетворяет уравнениям

$$
\begin{aligned}
j_{M} \circ P(\xi, \eta) & =-P\left(j_{L} \xi, \eta\right) \\
P(\xi, \eta)-P(\eta, \xi) & =P\left(j_{L} \xi, j_{L} \eta\right)-P\left(j_{L} \eta, j_{L} \xi\right)
\end{aligned}
$$

ЛЕмма 2. Любой тензор, удовлетворяющий системе (4), (5), может быть представлен в виде $(2)$, где $\Phi^{(2)}$ - симметричный тензор. 
ДоКАЗАТЕЛЬСТво. Поскольку любое билинейное отображение представимо в виде суммы $j_{L}-j_{M}$-линейного и антилинейного по первому аргументу отображений, из (4) следует, что

$$
P(\xi, \eta)=j_{M} \circ B(\xi, \eta)-B\left(j_{L} \xi, \eta\right)
$$

для некоторого тензора $B$. Необходимо показать, что отображение $B: T_{x} L \otimes T_{x} L \rightarrow T_{y} M$ можно выбрать симметричным. Рассмотрим разложение $B$ на симметричную и кососимметричную составляющие:

$$
B=B_{0}+B_{1}, \quad B_{i}(\xi, \eta)=\frac{1}{2}\left(B(\xi, \eta)+(-1)^{i} B(\eta, \xi)\right), \quad i=0,1 .
$$

Подставляя это выражение в (5) и учитывая (6), получим

$$
j_{M} \circ B_{1}(\xi, \eta)-B_{1}\left(j_{L} \xi, \eta\right)-B_{1}\left(\xi, j_{L} \eta\right)-j_{M} \circ B_{1}\left(j_{L} \xi, j_{L} \eta\right)=0 .
$$

Разложим $B_{1}$ на линейную и антилинейную по первому и второму аргументам составляющие:

$$
\begin{gathered}
B_{1}=B_{1}^{0,0}+B_{1}^{0,1}+B_{1}^{1,0}+B_{1}^{1,1}, \quad B_{1}^{1,1} \equiv 0, \\
B_{1}^{0,0}(\xi, \eta)=\frac{1}{2}\left(B_{1}(\xi, \eta)-B_{1}\left(j_{L} \xi, j_{L} \eta\right)\right), \\
B_{1}^{0,1}(\xi, \eta)=\frac{1}{2}\left(B_{1}(\xi, \eta)+j_{M} \circ B_{1}\left(\xi, j_{L} \eta\right)\right), \\
B_{1}^{1,0}(\xi, \eta)=\frac{1}{2}\left(B_{1}(\xi, \eta)+j_{M} \circ B_{1}\left(j_{L} \xi, \eta\right)\right) .
\end{gathered}
$$

С помощью (7) легко проверяются свойства

$$
B_{1}^{r, s}(\xi, \eta)=(-1)^{r+1} j_{M} \circ B_{1}^{r, s}\left(j_{L} \xi, \eta\right)=(-1)^{s+1} j_{M} \circ B_{1}^{r, s}\left(\xi, j_{L} \eta\right), \quad r, s=0,1 .
$$

Заметим, что тензор

$$
\widehat{B}_{0}(\xi, \eta)=B_{1}^{0,1}(\xi, \eta)-B_{1}^{1,0}(\xi, \eta)=\frac{j_{M}}{2}\left(B_{1}\left(\xi, j_{L} \eta\right)-B_{1}\left(j_{L} \xi, \eta\right)\right)
$$

симметричен, а тензор $B_{1}+\widehat{B}_{0}=B_{1}^{0,0}+2 B_{1}^{0,1}$ является $j_{L}-j_{M}$-линейным по $\xi$. Следовательно, преобразование $B=B_{0}+B_{1} \mapsto B-\left(B_{1}+\widehat{B}_{0}\right)=B_{0}-\widehat{B}_{0}$ определяет искомый симметричньй тензор $\Phi^{(2)}=B_{0}-\widehat{B}_{0}$, удовлетворяющий (2).

Итак, уравнение $\mathscr{E}^{2}$ выделяется как подуравнение в $\mathscr{E}^{1}$ соотношениями (3)-(5). При этом уравнение (4) является тождеством, поскольку

$$
j_{M} \circ \Phi=\Phi \circ j_{L}, \quad j_{L} \circ d j_{L}=-d j_{L} \circ\left(j_{L} \otimes \mathbf{1}\right), \quad j_{M} \circ d j_{M}=-d j_{M} \circ\left(j_{M} \otimes \mathbf{1}\right) .
$$

Подставляя уравнение (3) в (5), находим

$$
\begin{aligned}
& d j_{M}(\Phi \xi, \Phi \eta)-d j_{M}(\Phi \eta, \Phi \xi)-d j_{M}\left(j_{M} \Phi \xi, j_{M} \Phi \eta\right)+d j_{M}\left(j_{M} \Phi \eta, j_{M} \Phi \xi\right) \\
& =\Phi\left(d j_{L}(\xi, \eta)-d j_{L}(\eta, \xi)-d j_{L}\left(j_{L} \xi, j_{L} \eta\right)+d j_{L}\left(j_{L} \eta, j_{L} \xi\right)\right)
\end{aligned}
$$




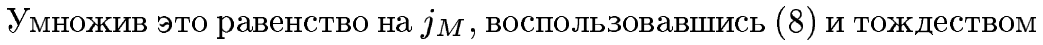

$$
N_{j}(\xi, \eta)=-d j(j \xi, \eta)-d j(\xi, j \eta)+d j(j \eta, \xi)+d j(\eta, j \xi)
$$

получим уравнение $N_{j_{M}} \circ \Phi^{\wedge 2}=\Phi \circ N_{j_{L}}$. Теорема доказана.

Итак, теорема 1 задает уравнения в точке (на $\left.T_{x} L\right)$, определяющие возможность построения псевдоголоморфного отображения на уровне 2-струй.

Введем линейное пространство тензоров Ниенхейса в комплексном линейном пространстве $\left(\mathbb{C}^{n}, j\right)$ :

$$
\mathcal{N}_{j}^{(n)}=\left\{N \in \wedge^{2}\left(\mathbb{C}^{n}\right)^{*} \otimes \mathbb{C}^{n} \mid N(j \xi, \eta)=-j N(\xi, \eta), N(\xi, \eta)=-N(\eta, \xi)\right\} .
$$

Рассмотрим на $\mathscr{N}_{j}^{(n)}$ отображение факторизации

$$
\theta: \mathscr{N}_{j}^{(n)} \rightarrow \mathscr{N}_{j}^{(n)} / \mathrm{Gl}_{\mathbb{C}}(n)
$$

сопоставляющее тензору его орбиту относительно полной групшы линейных комплексных преобразований. Подход Картана к формальной интегрируемости, а также подход Спенсера (см. теорему Гольдшмидта в [8], [6]) заключается в нахождении методом продолжений-проекций уравнения $\mathscr{E}(\infty) \subset \mathscr{E}^{2} \subset J_{\mathrm{PH}}^{1}$, допускающего продолжение в пространство 2-струй, символы которого образуют расслоение. В частности, надо требовать $\left(\mathscr{E}^{2}\right)_{x^{\prime}, y^{\prime}} \neq 0$ для всех $x^{\prime} \in L, y^{\prime} \in M$. Это будет так, если потребовать постоянства естественных отображений $\theta_{L}^{(N)}: \mathscr{O}_{L}(x) \rightarrow \mathscr{N}_{j_{L}}^{(l)} / \mathrm{Gl}_{\mathbb{C}}(l), \theta_{M}^{(N)}: \mathscr{O}_{M}(y) \rightarrow$ $\mathcal{N}_{j_{M}}^{(m)} / \mathrm{Gl}_{\mathbb{C}}(m)$, сопоставляющих точке $x$ образ тензора Ниенхейса $\left(N_{j}\right)_{x}$ в этой точке при отображении (11), и существования морфизма $\Phi: T_{x} L \rightarrow T_{y} M$, сопрягающего $N_{j_{L}}$ и $N_{j_{M}}$. Как показывает следующий пример, многообразия $\left(L, j_{L}\right)$ с $\theta_{L}^{(N)} \equiv$ const cyществуют.

ПримеР 1. Рассмотрим группу Ли $G$. Алгебра Ли группы $G \times G$ изоморфна $\mathscr{G} \oplus \mathscr{G}$. Введем почти комплексную структуру на $T_{e}(G \times G)$ формулой $j(\xi, \eta)=(-\eta, \xi)$ и продолжим ее левыми сдвигами на всю группу. Тензор Ниенхейса левоинвариантен и имеет вид

$$
\begin{gathered}
N((\xi, 0),(\eta, 0))=-N((0, \xi),(0, \eta))=(-[\xi, \eta],[\xi, \eta]) \\
N((\xi, 0),(0, \eta))=N((0, \xi),(\eta, 0))=([\xi, \eta],[\xi, \eta]) .
\end{gathered}
$$

Это показывает, что линейная классификация однородных тензоров Ниенхейса на комплексификациях алгебр Ли тесно связана с класификацией действительных алгебр Ли.

ЗАмЕчАниЕ 1 . В работе [10] показано, что на многообразии $J_{\mathrm{PH}}^{1}(L, M)$ существует каноническая почти комплексная структура $j^{[1]}$. Эта структура определяется как $\left(j_{L} \oplus j_{M}\right) \oplus j_{M}$ на $T_{p} J_{\mathrm{PH}}^{1}=T_{p} J_{\mathrm{PH}}^{0} \oplus \mathrm{Smbl}_{\mathscr{E} 1}^{1}$ в разложении, индуцированном минимальными связностями на многообразиях $L$ и $M$. По определению эти связности такие, что их формы кручения совпадают с формами кручения почти комплексных структур, т.е. с соответствующими тензорами Ниенхейса [11]. В точках $p \in \mathscr{E}^{2} \subset J_{\mathrm{PH}}^{1}$ почти комплексная структура $j^{[1]}$ на $T_{p} J_{\mathrm{PH}}^{1}$ может быть определена иначе. Действительно, 1-РН-струя 
$p$ продолжается до 2-РН-струи $p^{(2)}$, и $p^{(2)}$ определяет подпространство $L_{p^{(2)}}$ в касательном пространстве к $J_{\mathrm{PH}}^{1}$ (касательное пространство к порождающему $p^{(2)}$ грабику, ср. [7]). Таким образом, касательное пространство $T_{p} J_{\mathrm{PH}}^{1}$ изоморфно прямой сумме слоя проекции $\pi: J_{\mathrm{PH}}^{1}(L, M) \rightarrow L$ с почти комплексной структурой $j_{M}$ и пространства $L_{p^{(2)}}$ с почти комплексной структурой $j_{L}$. Ввиду комплексности обоих слагаемых на $T_{p} J_{\mathrm{PH}}^{1}$ имеется каноническая комплексная структура. Заметим, что хотя продолжение $p^{(2)}$ может быть построено неоднозначно, структура $j_{p}^{[1]}$ не зависит от него. Предположим, что подмножества $\left(\mathscr{E}^{2}\right)_{x^{\prime}, y^{\prime}}$ не зависят от точек $x^{\prime} \in L, y^{\prime} \in M$ и образуют расслоение над $J_{\mathrm{PH}}^{0}$, т.е. $\mathscr{E}^{2}-$ подмногообразие в $\mathscr{E}^{1}$. Из теоремы 1 следует, что это подмногообразие не является в общем случае комплексным, и это “измеряет" неинтегрируемость структур $j_{L}$ и $j_{M}$. Действительно, в силу антилинейности квадрика, выделяемая в комплексном линейном пространстве $\left\{\Phi \in T_{x}^{*} L \otimes T_{y} M \mid j_{M} \circ \Phi=\Phi \circ j_{L}\right\}$ условием $N_{j_{M}} \circ \Phi^{\wedge 2}=\Phi \circ N_{j_{L}}$, не является комплексным подмногообразием, если $\left.N_{j_{M}}\right|_{\operatorname{Im} \Phi^{\wedge 2}} \not \equiv 0$. Таким образом, на $J_{\mathrm{PH}}^{2}$ не существует почти комплексной структуры такой, что ограничение проекции $\pi_{\mathrm{PH}}^{2,1}: J_{\mathrm{PH}}^{2} \rightarrow J_{\mathrm{PH}}^{1}$ псевдоголоморфно.

Поскольку $J_{\mathrm{PH}}^{1}(L, M)$ обладает канонической почти комплексной структурой $j_{L, M}^{[1]}$, можно рассмотреть многообразие $J_{\mathrm{PH}}^{[2]}(L, M)=J_{\mathrm{PH}}^{1}\left(L, J_{\mathrm{PH}}^{1}(L, M)\right)$ с почти комплексной структурой $j_{L, M}^{[2]}$ и определить индуктивно многообразия

$$
J_{\mathrm{PH}}^{[k+1]}(L, M)=J_{\mathrm{PH}}^{1}\left(L, J_{\mathrm{PH}}^{[k]}(L, M)\right)
$$

с почти комплексной структурой $j_{L, M}^{[k+1]}$. Заметим, что $J_{\mathrm{PH}}^{k}(L, M) \subset J_{\mathrm{PH}}^{[k]}(L, M)$. Скажем, что 1-струя $\Phi \in\left(J_{\mathrm{PH}}^{1}(L, M)\right)_{x, y}$ поднимается до $k$-струи, если $\left(J_{\mathrm{PH}}^{k}\right)_{x, y} \supset$ $\left(\pi_{\mathrm{PH}}^{k, 1}\right)^{-1}(\Phi) \neq \varnothing$. В силу теоремы 1 1-струя $\Phi$ поднимается до 2-струи тогда и только тогда, когда $N_{j_{M}} \circ \Phi^{\wedge 2}=\Phi \circ N_{j_{L}}$; в этом случае существует (необязательно единственное) поднятие $\Phi^{[2]} \in\left(J_{\mathrm{PH}}^{2}\right)_{x, y} \subset\left(J_{\mathrm{PH}}^{[2]}\right)_{x, y}$, являющееся 1-струей в объемлющем пространстве. Эта 1-струя может быть поднята до 2-струи $\Phi^{[3]} \in\left(J_{\mathrm{PH}}^{[3]}\right)_{x, y}$ тогда и только тогда, когда $N_{j_{L, M}^{[1]}} \circ\left(\Phi^{[2]}\right)^{\wedge 2}=\Phi^{[2]} \circ N_{j_{L}}$. В общем случае, если среди поднятий $\Phi^{[k]} \in\left(J_{\mathrm{PH}}^{[k]}\right)_{x, y}$ струи $\Phi^{[k-1]} \in\left(J_{\mathrm{PH}}^{[k-1]}\right)_{x, y}$ имеется такое, для которого

$$
N_{j_{L, M}^{[k-1]}} \circ\left(\Phi^{[k]}\right)^{\wedge 2}=\Phi^{[k]} \circ N_{j_{L}}
$$

то струя $\Phi^{[k]} \in\left(J_{\mathrm{PH}}^{[k]}\right)_{x, y}$ может быть поднята до струи $\Phi^{[k+1]} \in\left(J_{\mathrm{PH}}^{[k+1]}\right)_{x, y}$. Итак, мы имеем ряд необходимых условий (12), при выполнении которых существуют поднятия $\Phi^{[r]} \in\left(J_{\mathrm{PH}}^{[r]}\right)_{x, y} 1$-струи $\Phi \in\left(J_{\mathrm{PH}}^{1}\right)_{x, y}$. Это дает возможность искать такие поднятия, для которых $\Phi^{[r]} \in\left(J_{\mathrm{PH}}^{r}\right)_{x, y}$.

\section{§ 2. Построение псевдоголоморфного отображения на уровне высших струй}

В $\S 1$ мы нашли алгебру инвариантов $\mathscr{A}_{j}^{2}=\left\langle j, N_{j}\right\rangle$, порожденную относительно коммутатора элементами $j, N_{j}$, такую, что $\mathscr{E}_{x, y}^{2}=\left\{\Phi: T_{x} L \rightarrow T_{y} M \mid \mathscr{A}_{j_{M}}^{2} \circ \Phi^{\wedge \star}=\Phi \circ \mathscr{A}_{j_{L}}\right\}$. Применяя метод продолжений-проекций (см. $\S 1)$, мы сужаем уравнение $\mathscr{E}^{2}$ до уравнения $\mathscr{E}^{\infty}$ и находим алгебру инвариантов $\mathscr{A}_{j}^{\infty}$ такую, что $\mathscr{E}_{x, y}^{\infty}=\left\{\Phi: T_{x} L \rightarrow T_{y} M \mid\right.$ 
$\left.\mathscr{A}_{j_{M}}^{\infty} \circ \Phi^{\wedge \star}=\Phi \circ \mathscr{A}_{j_{L}}^{\infty}\right\}$. Как показано в п. 6.2 (замечание 7$)$, ни алгебра $\mathscr{A}_{j}^{2}$, ни ее замыкание относительно дифференциальной скобки Ниенхейса (см. [12], [6]) - алгебра Ниенхейса $\mathscr{A}_{j}^{\mathcal{N}}$ - не совпадают с искомой алгеброй $\mathscr{A}_{j}^{\infty}$, т.е. $\mathscr{A}_{j}^{\mathcal{N}} \neq \mathscr{A}_{j}^{\infty}=\left\langle j, N_{j}, \ldots\right\rangle$. Ниже мы построим новьй инвариант, приходящий из пространств высших струй и являющийся новой (третьей) образуюшей $\mathscr{A}_{j}^{\infty}$. Отметим, что, проводя дальнейшие вычисления, можно указать и последующие образующие.

ТЕорема 2. Существуют новые тензорные инварианты - высшие тензоры Ниенхейса - такие, что $\mathscr{A}_{j}^{\infty}=\left\langle j, N_{j}, N_{j}^{(2)}, \ldots\right\rangle$. Первый из таких инвариантов $N_{j}^{(2)}$ имеет вид

$$
\begin{aligned}
N_{j}^{(2)}(\xi, \eta, \zeta, \nu)=- & {\left[N_{j}(\xi, \eta), j N_{j}(\zeta, \nu)\right]-\left[j N_{j}(\xi, \eta), N_{j}(\zeta, \nu)\right] } \\
& +N_{j}\left(\left[\xi, j N_{j}(\zeta, \nu)\right], \eta\right)+N_{j}\left(\xi,\left[\eta, j N_{j}(\zeta, \nu)\right]\right)+j N_{j}\left(\left[\xi, N_{j}(\zeta, \nu)\right], \eta\right) \\
& +j N_{j}\left(\xi,\left[\eta, N_{j}(\zeta, \nu)\right]\right)-N_{j}\left(\left[\zeta, j N_{j}(\xi, \eta)\right], \nu\right)-N_{j}\left(\zeta,\left[\nu, j N_{j}(\xi, \eta)\right]\right) \\
& -j N_{j}\left(\left[\zeta, N_{j}(\xi, \eta)\right], \nu\right)-j N_{j}\left(\zeta,\left[\nu, N_{j}(\xi, \eta)\right]\right),
\end{aligned}
$$

әде $\xi, \eta, \zeta, \nu$ - произвольные векторные поля, продолжающие векторы, заданные в mочке $x$.

В общем случае $N_{j}^{(2)}$ является первым среди образующих инвариантов из $\mathscr{A}_{j}^{\infty}$, различающим структуры $j$ и $-j$ (см. $§ 5$, замечание 4 ).

ДокАЗАТЕльство. Как показано в теореме 1 , для уравнения $\widetilde{\mathscr{E}}^{1}=\mathscr{E}^{2} \subset J_{\mathrm{PH}}^{1}$ проекции $\widetilde{\mathscr{E}}^{k}, \mathscr{E}^{k+1}$ совпадают. Поэтому вместо задачи о проекциях высших продолжений уравнения $\mathscr{E}^{1}$, ведущей к формально интегрируемому уравнению (см. 1 ), можно рассматривать задачу поиска проекции высших продолжений для нового уравнения $\widetilde{\mathscr{E}}^{1}$ и, как первый шаг, задачу о поднятии на один уровень. Решение на уровне 1-струй уравнения $\widetilde{\mathscr{E}}^{1}$ продолжается до решения на уровне 2-струй, если и только если вьполнены уравнения (1) и

$$
\begin{aligned}
U(\xi, \eta, \theta) & :=\Phi^{(2)}\left(N_{j_{L}}(\xi, \eta), \theta\right)-N_{j_{M}}\left(\Phi^{(2)}(\xi, \theta), \Phi \eta\right)-N_{j_{M}}\left(\Phi \xi, \Phi^{(2)}(\eta, \theta)\right) \\
& =d\left(N_{j_{M}}\right)(\Phi \xi, \Phi \eta, \Phi \theta)-\Phi \circ d\left(N_{j_{L}}\right)(\xi, \eta, \theta) .
\end{aligned}
$$

Из (1) и (14) имеем

$$
\begin{aligned}
U(\xi, & \left.\eta, j_{L} \theta\right)+j_{M} U(\xi, \eta, \theta)=2 j_{M} \circ \Phi^{(2)}\left(N_{j_{L}}(\xi, \eta), \theta\right)+d j_{M}\left(\Phi \theta, N_{j_{M}}(\Phi \xi, \Phi \eta)\right) \\
& -\Phi \circ d j_{L}\left(\theta, N_{j_{L}}(\xi, \eta)\right)-N_{j_{M}}\left(d j_{M}(\Phi \theta, \Phi \xi), \Phi \eta\right)-N_{j_{M}}\left(\Phi \xi, d j_{M}(\Phi \theta, \Phi \eta)\right) \\
& +\Phi \circ N_{j_{L}}\left(d j_{L}(\theta, \xi), \eta\right)+\Phi \circ N_{j_{L}}\left(\xi, d j_{L}(\theta, \eta)\right) \\
= & d\left(N_{j_{M}}\right)\left(\Phi \xi, \Phi \eta, j_{M} \Phi \theta\right)+j_{M} \circ d\left(N_{j_{M}}\right)(\Phi \xi, \Phi \eta, \Phi \theta) \\
& -\Phi \circ d\left(N_{j_{L}}\right)\left(\xi, \eta, j_{L} \theta\right)-\Phi \circ j_{L} \circ d\left(N_{j_{L}}\right)(\xi, \eta, \theta),
\end{aligned}
$$

откуда $2 j_{M} \circ \Phi^{(2)}\left(N_{j}(\xi, \eta), \theta\right)=R_{j_{M}}(\Phi \xi, \Phi \eta, \Phi \theta)-\Phi \circ R_{j_{L}}(\xi, \eta, \theta)$, где

$$
\begin{aligned}
R_{j}(\xi, \eta, \theta)= & d\left(N_{j}\right)(\xi, \eta, j \theta)+j d\left(N_{j}\right)(\xi, \eta, \theta) \\
& +N_{j}(d j(\theta, \xi), \eta)+N_{j}(\xi, d j(\theta, \eta))-d j\left(\theta, N_{j}(\xi, \eta)\right) .
\end{aligned}
$$

Так как $\Phi^{(2)}$ - симметричный тензор, для тензора

$$
N_{j}^{(2)}(\xi, \eta, \zeta, \nu)=R_{j}\left(\xi, \eta, N_{j}(\zeta, \nu)\right)-R_{j}\left(\zeta, \nu, N_{j}(\xi, \eta)\right)
$$


имеем

$$
N_{j_{M}}^{(2)} \circ\left(\Phi^{\wedge 2}\right)^{\wedge 2}=\Phi \circ N_{j_{L}}^{(2)} .
$$

Итак, согласно (17) мы имеем новьй инвариант $N_{j}^{(2)} \in \mathscr{A}_{j}^{3} \subset \mathscr{A}_{j}^{\infty}$, которьй будем назьвать высиим тензором Ниенхейса. Формула (16) показьвает тензорньй характер этого инварианта, тем самым, $N_{j}^{(2)} \in \wedge^{2}\left(\wedge^{2} T_{x}^{*}\right) \otimes T_{x}$, а формула (13), следующая из (14) и (15), показывает его независимость от евклидовой связности $\nabla$, определяющей $d=d_{\nabla}$. Возможно, тензор $N_{j}^{(2)}$ валентности $(1,4)$ мог бы оказаться зависимым с тензорами четвертого порядка, получаемьпи из $\mathscr{A}_{j}^{2}$, такими как $N_{j}\left(N_{j}(\xi, \eta), N_{j}(\zeta, \nu)\right)$, т.е. не являться новым, но следующий пример показывает, что это не так.

ПримеР 2. Пусть $\partial_{i}=\partial / \partial x^{i}$ - базисные векторы в $\mathbb{R}^{4}=\mathbb{R}^{4}\left(x^{1}, x^{2}, x^{3}, x^{4}\right)$. Зададим комплексную структуру соотношениями

$$
j \partial_{1}=\partial_{2}, \quad j \partial_{2}=-\partial_{1}, \quad j \partial_{3}=\partial_{4}+x^{2} \partial_{1}, \quad j \partial_{4}=-\partial_{3}-x^{2} \partial_{2} .
$$

Для тензоров $N_{j}$ и $N_{j}^{(2)}$ имеем

$$
\begin{gathered}
N_{j}\left(\partial_{1}, \partial_{2}\right)=0, \quad N_{j}\left(\partial_{3}, \partial_{4}\right)=x^{2} \partial_{1}, \quad N_{j}\left(\partial_{1}, \partial_{3}\right)=-N_{j}\left(\partial_{2}, \partial_{4}\right)=\partial_{1}, \\
N_{j}\left(\partial_{2}, \partial_{3}\right)=N_{j}\left(\partial_{1}, \partial_{4}\right)=-\partial_{2}, \quad N_{j}^{(2)}\left(\partial_{1}, \partial_{3}, \partial_{3}, \partial_{4}\right)=-\partial_{1}, \\
N_{j}^{(2)}\left(\partial_{1}, \partial_{3}, j \partial_{3}, \partial_{4}\right)=N_{j}^{(2)}\left(\partial_{1}, \partial_{3}, \partial_{4}, \partial_{4}\right)+x^{2} N_{j}^{(2)}\left(\partial_{1}, \partial_{3}, \partial_{1}, \partial_{4}\right)=0 .
\end{gathered}
$$

\section{§3. Критерий разрешимости уравнения Коши-Римана}

\section{и формальные нормальные формы почти комплексных структур}

3.1. Разрешимость уравнения Коши-Римана. Множество РН-струй может быть локально описано следующим образом:

$$
\begin{gathered}
J_{\mathrm{PH}}^{k}=\left\{\left(x, y, \Phi^{(1)}, \ldots, \Phi^{(k)}\right) \mid x \in L, y \in M, \Phi^{(i)} \in \operatorname{Smbl}_{\mathrm{PH}}^{i}(L, M) \subset \mathbb{S}^{i} T^{*} L \otimes T M\right\}, \\
\sum_{\substack{r_{1}+\cdots+r_{p}=k \\
1 \leqslant p \leqslant k}} \sum_{\substack{1 \leqslant i_{s}^{r} \leqslant k, \#\left\{i_{s}^{r}\right\}=k \\
i_{1}^{1}<i_{2}^{1}<\cdots<i_{p}^{1} \\
i_{s}^{1}<i_{s}^{2}<\cdots<i_{s}^{r}}} d^{p-1} j_{M}\left(\Phi^{\left(r_{1}\right)}\left(\xi_{i_{1}^{1}}, \ldots, \xi_{i_{1}^{r_{1}}}\right), \ldots, \Phi^{\left(r_{p}\right)}\left(\xi_{i_{p}^{1}}, \ldots, \xi_{i_{p}}\right)\right) \\
=\sum_{\substack{1 \leqslant p \leqslant k, i_{1}^{1}=1,1 \leqslant i_{s}^{r} \leqslant k, \#\left\{i_{s}^{r}\right\}=k, i_{s}^{1}<i_{s}^{2}<\cdots<i_{s}^{*}}} \Phi^{(k-p+1)}\left(d j_{L}^{p-1}\left(\xi_{i_{1}^{1}}, \ldots, \xi_{i_{1}^{p}}\right), \xi_{i_{2}^{1}}, \ldots, \xi_{i_{2}^{k-p}}\right) .
\end{gathered}
$$

Здесь \# $S$ обозначает количество различных элементов множества $S$.

Следующая теорема обобщает теорему 1.

Теорема 3. Пусть отображсение $u: \mathscr{O}_{L}(x) \rightarrow \mathscr{O}_{M}(y)$ таково, что

$$
j_{M} \circ u_{*}=u_{*} \circ j_{L}\left(\bmod \mu^{k-1}\right), \quad N_{j_{M}} \circ u_{*}^{\wedge 2} \equiv u_{*} \circ N_{j_{L}}\left(\bmod \mu^{k-1}\right), \quad 2 \leqslant k \leqslant \infty .
$$

Тогда существует отображсение $\widetilde{u}: \mathscr{O}_{L}(x) \rightarrow \mathscr{O}_{M}(y)$ mакое, что $j_{M} \circ \widetilde{u}_{*}-\widetilde{u}_{*} \circ j_{L} \in \mu^{k}$, $u_{*}-\widetilde{u}_{*} \in \mu^{k-1}$. В частности, если $\left(u_{*}\right)_{x} \neq 0$, то $\left(\widetilde{u}_{*}\right)_{x} \neq 0, u$ если $\left(u_{*}\right)_{x}-$ вложсение или отображсние "на", то это жсе можно сказать и об $\left(\widetilde{u}_{*}\right)_{x}$. 
ДокАЗАТЕЛЬСтво. Отметим, что $(k-1)$-РН-струя $\left(\Phi, \Phi^{(2)}, \ldots, \Phi^{(k-1)}\right)$ поднимается до $k$-РН-струи тогда и только тогда, когда для некоторого симметричного тензора $\Phi^{(k)}$ вьполнено уравнение (18). Рассмотрим тензор

$$
P_{k}=j_{M} \circ \Phi^{(k)}-\Phi^{(k)} \circ\left(j_{L} \otimes \mathbf{1}_{L}^{k-1}\right) .
$$

Далее нам потребуются две вспомогательные леммы.

ЛЕмма 3. Для всякого $k \geqslant 1$ тензор $P_{k}$, определенный равенством (19), удовлетворяет следующим свойствам:

$$
\begin{gathered}
j_{M} \circ P_{k}=-P_{k} \circ\left(j_{L} \otimes \mathbf{1}_{L}^{k-1}\right) \\
P_{k}\left(\xi, \eta, \vec{\theta}^{k-2}\right)-P_{k}\left(\eta, \xi, \vec{\theta}^{k-2}\right)=P_{k}\left(j_{L} \xi, j_{L} \eta, \vec{\theta}^{k-2}\right)-P_{k}\left(j_{L} \eta, j_{L} \xi, \vec{\theta}^{k-2}\right) \\
P_{k} \in T_{x}^{*} L \otimes \mathbb{S}^{k-1} T_{x}^{*} L \otimes T_{y} M
\end{gathered}
$$

Лемма 4. Любой тензор $P_{k}$, удовлетворяющий условиям (20)-(22), может быть представлен в виде $(19)$, где $\Phi^{(k)} \in \mathbb{S}^{k} T_{x}^{*} L \otimes T_{y} M$.

ДоказАтельство. Рассмотрим тензор $B\left(\xi, \vec{\eta}^{(k-1)}\right)$ такой, что $P_{k}=j_{M} \circ B-B$ 。 $\left(j_{L} \otimes \mathbf{1}_{L}^{k-1}\right)$. В силу (20) его всегда можно найти, а согласно (22) можно считать, что $B$ симметричен по последним $k-1$ аргументам: $B \in T_{x}^{*} L \otimes \mathbb{S}^{k-1} T_{x}^{*} L \otimes T_{y} M$. Заметим, что при изменении $B$ на $j_{L}-j_{M}$-линейный по первому аргументу тензор соответствующий тензор $P_{k}$ не меняется. Таким образом, в разложении $B=B^{0, *}+B^{1, *}$ на $j_{L}-j_{M}$-линейную и антилинейную по первому аргументу компоненты можно избавиться от первого слагаемого и считать $B=B^{1, *}$.

Рассмотрим разложение

$$
B^{1, *}=\sum_{p=0}^{k-1} C_{p}, \quad C_{p}=\sum_{\tau_{p} \in \mathbb{Z}_{2}^{k-1}} B^{1, \tau_{p}}, \quad \mathbb{Z}_{2}=\{0,1\}
$$

количество единиц в $\tau_{p}$ равно $\#_{1} \tau_{p}=p$, и тензор $B^{1, \tau_{p}}$ является $j_{L}-j_{M}$-линейным или антилинейным по $s$-му аргументу, если на $s$-м месте в $\left(1, \tau_{p}\right)$ стоит соответственно 0 или 1. Заметим, что если единицы образуют в $\tau_{p}$ множество $i_{1}, \ldots, i_{r}$, а нули - множество $j_{1}, \ldots, j_{k-r-1}$, то $B^{1, \tau_{p}}$ симметричен по соответствуюшим аргументам $\xi_{i_{1}}, \ldots, \xi_{i_{r}}$ и $\xi_{j_{1}}, \ldots, \xi_{j_{k-r-1}}$. Заметим также, что тензор $B^{1, \tau_{p}}$ симметричен по аргументам $\xi_{1}, \xi_{i_{s}}$. Например, для $B^{1,1, *}$, пользуясь $j_{L}-j_{M}$-антилинейностью по $\xi_{1}$, из формулы (21) имеем

$$
B^{1, *}\left(\xi, \eta, \vec{\theta}^{k-2}\right)+j_{M} B^{1, *}\left(\xi, j_{L} \eta, \vec{\theta}^{k-2}\right)=B^{1, *}\left(\eta, \xi, \vec{\theta}^{k-2}\right)+j_{M} B^{1, *}\left(\eta, j_{L} \xi, \vec{\theta}^{k-2}\right)
$$

т.e. $B^{1,1, *}\left(\xi, \eta, \vec{\theta}^{k-2}\right)=B^{1,1, *}\left(\eta, \xi, \vec{\theta}^{k-2}\right)$.

Итак, тензор $B^{1, \tau_{p}}$ симметричен по аргументам $\xi_{1}, \xi_{i_{1}}, \ldots, \xi_{i_{r}}$. Несложно показать, что если $\gamma \in S_{k-1}-$ перестановка, то тензор $B^{1, \gamma\left(\tau_{p}\right)}$ совпадает с $B^{1, \tau_{p}} \circ(\mathbf{1} \times \gamma)$, где $\mathbf{1} \times \gamma$ действует как перестановка на аргументах; в случае $\gamma\left(\tau_{p}\right)=\tau_{p}$ утверждение совпадает со свойством симметричности $B^{1, \tau_{p}}$, отмеченным выше. Итак, если к тензору $C_{p}$ добавить сумму

$$
\sum_{\#_{1} \tau_{p+1}=p+1} B^{0, \tau_{p+1}}
$$


где $B^{0, \tau_{p+1}}=B^{1, \tau_{p}}$ о $\sigma$ и перестановка $\sigma \in S_{k}$ такова, что $\left(0, \tau_{p+1}\right)=\sigma^{-1}\left(1, \tau_{p}\right)$, то получится новый симметричньй тензор $\widehat{C}_{p} \in \mathbb{S}^{k} T_{x}^{*} L \otimes T_{y} M$.

Рассмотрим тензор

$$
\Phi^{(k)}=\sum_{p=0}^{k-1} \widehat{C}_{p},
$$

симметричный по построению, при этом $\Phi^{(k)}-B$ является $j_{L}-j_{M}$-линейным. Следовательно, тензор $P_{k}=j_{M} \circ \Phi^{(k)}-\Phi^{(k)} \circ\left(j_{L} \otimes \mathbf{1}^{k-1}\right)$, построенный по $\Phi^{(k)}$, совпадает с аналогичньм тензором, построенньм по $B$, и лемма 4 доказана.

Заметим, что предложенная конструкция несколько отличается от конструкции леммы $2(k=2)$. Для случая $k=3$ имеем $B^{1, *}=B^{1,0,0}+\left(B^{1,0,1}+B^{1,1,0}\right)+B^{1,1,1}$. Тензор $B^{1,1,1}$ симметричен, а $B^{1,0,0}$ симметричен по последним двум аргументам $(\eta, \theta)$. Следовательно, если $\sigma$ - циклическая перестановка аргументов, $\sigma(\xi, \eta, \theta)=(\eta, \theta, \xi)$, то $B^{1,0,0} \circ \sigma$ и $B^{1,0,0} \circ \sigma^{2}$ являются $j_{L}-j_{M}$-линейными по первому аргументу, а тензор $B^{1,0,0}+B^{1,0,0} \circ \sigma+B^{1,0,0} \circ \sigma^{2}$ симметричным. Тензор $B^{1,1,0}$ симметричен по аргументам $(\xi, \eta)$ в силу $(21)$ при $k=3$. Значит, $B^{1,1,0}(\xi, \eta, \theta)=A(\xi, \eta, \theta)+A(\eta, \xi, \theta)$ для некоторого тензора $A, j_{L}-j_{M}$-антилинейного по первым двум и линейного по последнему аргументам. Кроме того, тензор $B^{1, *}$ инвариантен относительно перестановки $\tau$ последних двух аргументов, $\tau(\xi, \eta, \theta)=(\xi, \theta, \eta)$. Поэтому

$$
\begin{aligned}
& B^{1,0,1}(\xi, \eta, \theta)=B^{1,1,0} \circ \tau(\xi, \eta, \theta)=A(\xi, \theta, \eta)+A(\theta, \xi, \eta) \\
& B^{1,0,1}(\xi, \eta, \theta)=B^{1,1,0}(\xi, \theta, \eta)=B^{1,1,0}(\theta, \xi, \eta)=B^{1,1,0} \circ \sigma^{2}(\xi, \eta, \theta) .
\end{aligned}
$$

Введем тензор

$$
B^{0,1,1}(\xi, \eta, \theta):=B^{1,1,0} \circ \sigma(\xi, \eta, \theta)=A(\eta, \theta, \xi)+A(\theta, \eta, \xi) .
$$

Заметим, что тензор $B^{1,1,0}+B^{1,0,1}+B^{0,1,1}$ симметричен, а $B^{0,1,1}$ является $j_{L}-j_{M}$-линейньм по первому аргументу. Таким образом, выполнено

$$
P_{3}=j_{M} \circ B^{1, *}-B^{1, *} \circ\left(j_{L} \otimes \mathbf{1} \otimes \mathbf{1}\right)=j_{M} \circ \Phi^{(3)}-\Phi^{(3)} \circ\left(j_{L} \otimes \mathbf{1} \otimes \mathbf{1}\right),
$$

где $\Phi^{(3)} \in \mathbb{S}^{3} T_{x}^{*} L \otimes T_{y} M$ определяется равенством

$$
\Phi^{(3)}=\left(B^{1,0,0}+B^{1,0,0} \circ \sigma+B^{1,0,0} \circ \sigma^{2}\right)+\left(B^{1,1,0}+B^{1,0,1}+B^{0,1,1}\right)+B^{1,1,1} .
$$

Леммы 3, 4 можно трактовать как точность следующего комплекса:

$$
0 \longrightarrow \mathscr{G}_{k} \stackrel{i}{\longrightarrow} \mathbb{S}^{k} T^{*} L \otimes T M \stackrel{\sigma_{k}}{\longrightarrow} T^{*} L \otimes \mathbb{S}^{k-1} T^{*} L \otimes T M \stackrel{\varkappa_{k} \oplus \nu_{k}}{\longrightarrow} \Theta_{k} \oplus \Delta_{k} \longrightarrow 0 .
$$

Здесь $\mathscr{G}_{k}$-продолжение символа $\mathscr{E}^{1}$ (см. [6], [7]) уравнения Коши-Римана, $\mathbb{S}^{k} T^{*} L \otimes T M-$ пространство всех символов, $i$ - включение, $\sigma_{k}$ - линеаризация оператора $\bar{\partial}: \sigma_{k}(\Psi)=$ $j_{M} \circ \Psi-\Psi \circ\left(j_{L} \otimes \mathbf{1}^{k-1}\right)$. Отображения $\varkappa_{k}$ и $\nu_{k}$ в пространства

$$
\Theta_{k}=\wedge^{2} T^{*} L \otimes \mathbb{S}^{k-2} T^{*} L \otimes T M \quad \text { и } \quad \Delta_{k}=\mathbb{S}^{k-1} T^{*} L \otimes \operatorname{Hom}_{\mathbb{C}}(T L, T M)
$$

имеют вид $\varkappa_{k}=\delta_{k}$ 。 $\widetilde{\sigma}_{k}$ и $\nu_{k}\left(X \otimes \vec{Y}^{(k-1)} \otimes Z\right)=\vec{Y}^{(k-1)} \otimes \varepsilon(X \otimes Z)$, где $\delta_{k}$ - оператор альтернирования по первым двум аргументам, $\delta_{k}(B)\left(X, Y, \vec{Z}^{(k-2)}\right)=B\left(X, Y, \vec{Z}^{(k-2)}\right)-$ $B\left(Y, X, \vec{Z}^{(k-2)}\right), \widetilde{\sigma}_{k} \Psi=j_{M} \circ \Psi-\Psi \circ\left(\mathbf{1} \otimes j_{L} \otimes \mathbf{1}^{k-2}\right)$, a $\varepsilon(B)=j_{M} \circ B+B \circ j_{L}-$ линеаризация оператора $\partial$. 
Заметим, что член $\Theta_{k}$, соответствуюший условию (21), дает условие сохранения дифференциала тензора Ниенхейса, а член $\Delta_{k}$, отвечающий условию (20), - тождество. Более подробно, выразим при помощи формулы (18) тензор $P_{k}$ через локальные представители $(k-1)$-струи $\left(\Phi, \Phi^{(2)}, \ldots, \Phi^{(k-1)}\right)$ отображения $u: \mathscr{O}_{L}(x) \rightarrow \mathscr{O}_{M}(y)$ и подставим соответствующее выражение в формулы леммы 3 . При этом условия (20) и (22) дадут тождество, а $(21)$ - условие сохранения $(k-1)$-струи тензора Ниенхейса. Соответствующие выкладки проводятся как и в теореме 1 для $k=2$.

Например, при $k=3$ имеем

$$
\begin{aligned}
P_{3}(\xi, \eta, \theta)= & j_{M} \Phi^{(3)}(\xi, \eta, \theta)-\Phi^{(3)}\left(j_{L} \xi, \eta, \theta\right) \\
= & \Phi d^{2} j_{L}(\xi, \eta, \theta)-d^{2} j_{M}(\Phi \xi, \Phi \eta, \Phi \theta)+\Phi^{(2)}\left(d j_{L}(\xi, \theta), \eta\right)+\Phi^{(2)}\left(d j_{L}(\xi, \eta), \theta\right) \\
& -d j_{M}\left(\Phi^{(2)}(\xi, \theta), \Phi \eta\right)-d j_{M}\left(\Phi \xi, \Phi^{(2)}(\eta, \theta)\right)-d j_{M}\left(\Phi^{(2)}(\xi, \eta), \Phi \theta\right) .
\end{aligned}
$$

Подстановка выражения (23) в (20) и (22) при $k=3$ дает тождество согласно (8) и формуле

$$
d^{2} j(j \xi, \eta, \theta)=-j d^{2} j(\xi, \eta, \theta)-d j(d j(\xi, \theta), \eta)-d j(d j(\xi, \eta), \theta) .
$$

Подставим выражение (23) в (21) при $k=3$ и воспользуемся формулами (1), (8), (24) и тождеством

$$
\begin{aligned}
& d j\left(N_{j}(\xi, \eta), \theta\right)+j d N_{j}(\xi, \eta, \theta)=d^{2} j(j \xi, j \eta, \theta)-d^{2} j(j \eta, j \xi, \theta)-d^{2} j(\xi, \eta, \theta)+d^{2} j(\eta, \xi, \theta) \\
& \quad+d j(d j(\xi, \theta), j \eta)-d j(d j(\eta, \theta), j \xi)+d j(j \xi, d j(\eta, \theta))-d j(j \eta, d j(\xi, \theta)),
\end{aligned}
$$

которое получается дифференцированием тождества (9). Имеем

$$
\begin{aligned}
0= & \left.\Phi^{(2)}\left(d j_{L}(\xi, \theta), \eta\right)+\Phi^{(2)}\left(d j_{L}(\xi, \eta), \theta\right)\right)-d j_{M}\left(\Phi^{(2)}(\xi, \theta), \Phi \eta\right)-d j_{M}\left(\Phi \xi, \Phi^{(2)}(\eta, \theta)\right) \\
& -d j_{M}\left(\Phi^{(2)}(\xi, \eta), \Phi \theta\right)+\Phi d^{2} j_{L}(\xi, \eta, \theta)-d^{2} j_{M}(\Phi \xi, \Phi \eta, \Phi \theta)-\Phi^{(2)}\left(d j_{L}(\eta, \theta), \xi\right) \\
& -\Phi^{(2)}\left(d j_{L}(\eta, \xi), \theta\right)+d j_{M}\left(\Phi^{(2)}(\eta, \theta), \Phi \xi\right)+d j_{M}\left(\Phi \eta, \Phi^{(2)}(\xi, \theta)\right) \\
& +d j_{M}\left(\Phi^{(2)}(\eta, \xi), \Phi \theta\right)-\Phi d^{2} j_{L}(\eta, \xi, \theta)+d^{2} j_{M}(\Phi \eta, \Phi \xi, \Phi \theta) \\
& -\Phi^{(2)}\left(d j_{L}\left(j_{L} \xi, \theta\right), j_{L} \eta\right)-\Phi^{(2)}\left(d j_{L}\left(j_{L} \xi, j_{L} \eta\right), \theta\right)+d j_{M}\left(\Phi^{(2)}\left(j_{L} \xi, \theta\right), j_{M} \Phi \eta\right) \\
& +d j_{M}\left(j_{M} \Phi \xi, \Phi^{(2)}\left(j_{L} \eta, \theta\right)\right)+d j_{M}\left(\Phi^{(2)}\left(j_{L} \xi, j_{L} \eta\right), \Phi \theta\right)-\Phi d^{2} j_{L}\left(j_{L} \xi, j_{L} \eta, \theta\right) \\
& +d^{2} j_{M}\left(j_{M} \Phi \xi, j_{M} \Phi \eta, \Phi \theta\right)+\Phi^{(2)}\left(d j_{L}\left(j_{L} \eta, \theta\right), j_{L} \xi\right)+\Phi^{(2)}\left(d j_{L}\left(j_{L} \eta, j_{L} \xi\right), \theta\right) \\
& -d j_{M}\left(\Phi^{(2)}\left(j_{L} \eta, \theta\right), j_{M} \Phi \xi\right)-d j_{M}\left(j_{M} \Phi \eta, \Phi^{(2)}\left(j_{L} \xi, \theta\right)\right)-d j_{M}\left(\Phi^{(2)}\left(j_{L} \eta, j_{L} \xi\right), \Phi \theta\right) \\
& +\Phi d^{2} j_{L}\left(j_{L} \eta, j_{L} \xi, \theta\right)-d^{2} j_{M}\left(j_{M} \Phi \eta, j_{M} \Phi \xi, \Phi \theta\right) \\
= & j_{M}\left(-\Phi^{(2)}\left(N_{j_{L}}(\xi, \eta), \theta\right)-\Phi d N_{j_{L}}(\xi, \eta, \theta)+N_{j_{M}}\left(\Phi^{(2)}(\xi, \theta), \Phi \eta\right)\right. \\
& \left.+N_{j_{M}}\left(\Phi \xi, \Phi^{(2)}(\eta, \theta)\right)+d N_{j_{M}}(\Phi \xi, \Phi \eta, \Phi \theta)\right) .
\end{aligned}
$$

Обращение в 0 последнего выражения в точности означает, что 1-струя из $\widetilde{\mathscr{E}}^{1}$ может быть продолжена до 2-струи.

По причине громоздкости мы опускаем выкладки для случая произвольного $k$. Теорема 2 доказана.

СлЕДСТВИЕ. Пусть тензоры Ниенхейса являются малыми порядка $k$ в точках $x \in L$ u $y \in M$, m.е. $N_{j_{L}} \in \mu_{x}^{k}, N_{j_{M}} \in \mu_{y}^{k}(1 \leqslant k \leqslant \infty)$. Тогда существует отображение $u: \mathscr{O}_{L}(x) \rightarrow \mathscr{O}_{M}(y)$ maкое, что $d u_{x} \neq 0 u j_{M} \circ u_{*}-u_{*} \circ j_{L} \in \mu^{k+1}$. 
ЗАмечание 2 . В случае $\operatorname{dim}_{\mathbb{C}} L=\operatorname{dim}_{\mathbb{C}} M=n, k=\infty$, это утверждение является формальной частью теоремы Ньюландера-Ниренберга (см. [1], [4]): если тензоры Ниенхейса почти комплексных структур $j$ и $j_{0}$ совпадают, то эти структуры локально изоморфны; здесь $j_{0}$ - стандартная комплексная структура на $\mathscr{O}_{\mathbb{C}}(0)\left(\right.$ конечно, $N_{j_{0}} \equiv 0$, см. формулу (9)). Обобщением этого факта является теорема 6 (см. $\S 5$ ). Заметим также, что из формальной эквивалентности не следует гладкая. Действительно, можно построить почти комплексную структуру $j$ такую, что $N_{j} \in \mu^{\infty}$, но $N_{j} \not \equiv 0$ (см. доказательство теоремы 5 в $\S 4$ ). Тогда $j$ формально эквивалентна $j_{0}$, но не эквивалентна гладко.

ЗАмЕЧАнИЕ 3 . Условия следствия можно сформулировать в виде $d N_{j_{L}} \in \mu_{x}^{k-1}$, $d N_{j_{M}} \in \mu_{y}^{k-1}$, где $d=d_{\nabla}$, a $\nabla$ - тривиальная связность. Заметим, однако, что не существует почти комплексных структур с условием $d N_{j} \equiv 0$, отличных от комплексных. Действительно, в этом случае существуют координаты, в которых компоненты тензора Ниенхейса являются постоянными, причем можно считать, что индуцированные координаты в фиксированном касательном пространстве $T_{x} \simeq \mathbb{C}^{n}$ являются комплексньми. Запишем действие почти комплексной структуры на базисные векторы $\partial_{r}=\partial / \partial x^{r}$ :

$$
j \partial_{2 r-1}=\partial_{2 r}+\sum a_{2 r}^{i} \partial_{i}, \quad j \partial_{2 r}=-\partial_{2 r-1}+\sum a_{2 r-1}^{i} \partial_{i}, \quad a_{t}^{s} \in \mu_{x}
$$

Если для почти любого вектора $\xi$ вьполнено $\operatorname{dim} N_{j}\left(\xi, T_{x}\right)=2 n-2$, т.е. $N_{j}$ - тензор общего вида, $\mathfrak{n}_{N_{j}}=2$ почти всюду (в смысле определения 4 из $\S 4$ ), то из условий $N_{j}\left(j \partial_{2 r-1}, \partial_{2 r}\right) \equiv N_{j}\left(j \partial_{2 s-1}, \partial_{2 s}\right) \equiv 0$ следует, что $a_{2 s}^{2 r} \equiv a_{2 s-1}^{2 r} \equiv a_{2 s}^{2 r-1} \equiv a_{2 s-1}^{2 r-1} \equiv 0$ для всех $r \neq s$, иными словами, $N_{j}\left(\partial_{2 r}, \partial_{2 s}\right)=0,-$ противоречие. Предположим теперь, что существует подпространство $K_{1} \subset T_{x}\left(\operatorname{dim} K_{1} \geqslant 4\right)$ такое, что $\left.N_{j}\right|_{\wedge^{2} K_{1}} \equiv 0$, причем будем считать $K_{1}$ максимальным, удовлетворяющим этому свойству. Продолжив $K_{1}$ аффинными сдвигами в фиксированной системе координат на окрестность точки $x$, получаем слоение $\mathscr{K}_{1}, \operatorname{dim} \mathscr{K}_{1}=\operatorname{dim} K_{1}, j$-инвариантное в силу $N_{j}(j \xi, \eta)=-j N_{j}(\xi, \eta)=0$ для $\xi, \eta \in T_{p} \mathscr{K}_{1}$, ограничение $j$ на слои которого является интегрируемым, $N_{j} \mid \mathscr{K}_{1} \equiv 0$. В дополнительном подпространстве выберем максимальное вырожденное относительно $N_{j}$ подпространство $K_{2}$, построим по нему слоение $\mathscr{K}_{2}$, и т.д. В итоге мы получим, что почти комплексная структура $j$ в окрестности точки $x$ представима как прямая сумма комплексных структур на слоях стандартных слоений $\mathscr{K}_{i}$, полученных из компонент комплексного разложения $T_{x}=\bigoplus K_{i}$. Теперь несложно получить противоречие с условием $d N_{j} \equiv 0$, если $j$ неинтегрируема в целом.

Заметим, что существуют нетривиальные связности $\nabla$ такие, что $d \nabla N_{j} \equiv 0$. Действительно, если в качестве $\nabla$ взять почти комплексную связность $[11]$, то $d \nabla j \equiv 0$, откуда $d_{\nabla} N_{j} \equiv 0$. В частности, такому свойству будет удовлетворять минимальная связность $\nabla$, которая несимметрична и имеет тензор кручения $T_{\nabla}=\frac{1}{4} N_{j}$ (такие связности всегда существуют, см. [11]).

3.2. Формальные нормальные формы почти комплексных структур. С помощью теоремы 3 можно построить формальные нормальные формы почти комплексныx структур, рассматривая почти комплексные структуры $\bmod \mu^{k}$, т.е. автоморфизмы $j: T_{x^{\prime}} X \rightarrow T_{x^{\prime}} X, x^{\prime} \in X$, такие, что $j^{2}=-\mathbf{1}_{X}\left(\bmod \mu_{x}^{k}\right)$.

Предположим, что мы построили нормальные формы почти комплексных структур $\bmod \mu^{k}: \mathscr{J}_{k}=\left\{j_{\lambda_{1}, \ldots, \lambda_{k-1}}\right\}$. Здесь $\lambda_{1}, \ldots, \lambda_{k-1}-$ параметры, задающие элементы $\mathscr{J}_{k}$. Рассмотрим те из них, которые продолжаются до почти комплексных структур $\bmod \mu^{k+1}$, т.е. те $J_{k} \in \mathscr{J}_{k}$, для которых существует автоморфизм касательного расслоения $\Delta J_{k} \in \mu^{k}$ с почти комплексной $\bmod \mu^{k+1}$ структурой $J_{k}+\Delta J_{k}$. 
Назовем такие структуры $k$-допустимыми. Если $J_{k}$ - почти комплексная структура $\bmod \mu^{k+1}$ и $\Delta J_{k} \in \mu^{k}$, то $J_{k}+\Delta J_{k}$ является почти комплексной структурой $\bmod \mu^{k+1}$ тогда и только тогда, когда $j_{0} \circ \Delta J_{k}+\Delta J_{k} \circ j_{0} \in \mu^{k+1}$, где $j_{0}=J_{k}(\bmod \mu)-$ линеаризация структуры $J_{k}$.

ПРЕДЛОЖЕНИЕ 1. Две почти комплексные $\bmod \mu^{k+1}(k \geqslant 1)$ структуры $J_{k} u$ $J_{k}+\Delta J_{k}$ эквивалентны $\mu^{k+1}$ тогда и только тогда, когда автоморфизм $\Delta J_{k}$ согласован с комплексной структурой $j_{0}$ по модулю $\mu^{k+1}$ :

$$
\begin{aligned}
N_{\left(j_{0}, \Delta J_{k}\right)}(\xi, \eta):= & {\left[j_{0} \xi, \Delta J_{k} \eta\right]+\left[\Delta J_{k} \xi, j_{0} \eta\right]-j_{0}\left[\xi, \Delta J_{k} \eta\right] } \\
& -j_{0}\left[\Delta J_{k} \xi, \eta\right]-\Delta J_{k}\left[\xi, j_{0} \eta\right]-\Delta J_{k}\left[j_{0} \xi, \eta\right] \in \mu^{k+1} .
\end{aligned}
$$

Действительно, $N_{\left(J_{k}+\Delta J_{k}, J_{k}\right)}:=N_{\left(J_{k}+\Delta J_{k}\right)}-N_{J_{k}} \equiv N_{\left(j_{0}, \Delta J_{k}\right)}\left(\bmod \mu^{k+1}\right)$.

Таким образом, $k$-допустимые почти комплексные структуры $j_{\lambda_{1}, \ldots, \lambda_{k-1}}$ из $\mathscr{J}_{k}$ определяют нормальные формы почти комплексных $\bmod \mu^{k+1}$ структур $j_{\lambda_{1}, \ldots, \lambda_{k}}$ из $\mathscr{J}_{k+1}$, где параметр $\lambda_{k}$ принимает значения в символах $k$-струй тензоров совместности Ниенхейса:

$$
\lambda_{k}=\left[N_{\left(j_{0}, \Delta J_{k}\right)}\right]_{k} \in\left(\mu^{k} \wedge^{2} T_{x}^{*} \otimes T_{x}\right) /\left(\mu^{k+1} \wedge^{2} T_{x}^{*} \otimes T_{x}\right)=\wedge^{2} T_{x}^{*} \otimes \mathbb{S}^{k} T_{x}^{*} \otimes T_{x} .
$$

Заметим, что $\lambda_{k}$ принимает значения в пространстве $\Theta_{k+2}$, которое возникает в доказательстве теоремы 3 .

Итак, мы построили нормальные формы почти комплексных $\bmod \mu^{k+1}$ структур: $\mathscr{J}_{k+1}=\left\{j_{\lambda_{1}, \ldots, \lambda_{k}}\right\}$, т.е. нашли структуры такие, что для любой почти комплексной $\bmod \mu^{k+1}$ структуры $j: T \mathscr{O}(x) \rightarrow T \mathscr{O}(x)$ существуют диффеоморфизм $\varphi_{k}: \mathscr{O}(x) \rightarrow \mathscr{O}(x)$ и набор параметров $\lambda_{1}, \ldots, \lambda_{k}$, удовлетворяющие тождеству $\varphi_{k}^{*} j \equiv j_{\lambda_{1}, \ldots, \lambda_{k}}\left(\bmod \mu^{k+1}\right)$.

Назовем деревом почти комплексных структур иерархию $\mathscr{J}_{1}=\left\{j_{0}\right\}, \mathscr{J}_{2}=\left\{j_{\lambda_{1}}\right\}$, $\mathscr{J}_{3}=\left\{j_{\lambda_{1}, \lambda_{2}}\right\}$, и т.д. Назовем ветвью дерева произвольную последовательность продолжающих друг друга почти комплексных структур из дерева $j_{0}, j_{\lambda_{1}}, j_{\lambda_{1}, \lambda_{2}}, j_{\lambda_{1}, \lambda_{2}, \lambda_{3}}$,

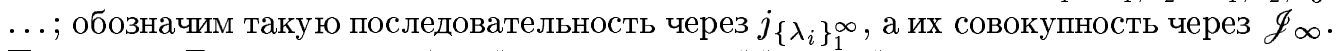
По теореме Бореля для любого формального диффеоморфизма существует индуцирующий его гладкий диффеоморфизм. Таким образом, получаем следующее утверждение.

ТЕорема 4. Множсество $\mathscr{J}_{\infty}$ задает формальные нормальные формы почти комплексных структур $\bmod \mu^{\infty}$, т.е. для любой формальной почти комплексной

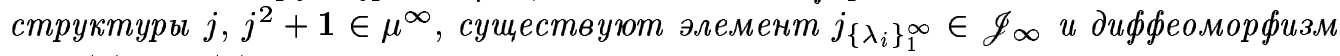
$\varphi: \mathscr{O}(x) \rightarrow \mathscr{O}(x)$ такие, что

$$
\varphi^{*} j \equiv j_{\left\{\lambda_{i}\right\}_{1}^{\infty}}\left(\bmod \mu^{\infty}\right)
$$

Заметим, что построенные нормальные формы не будут минимальными в смысле разбиения на непересекающиеся классы даже $\bmod \mu^{2}$, т.е. по модулю классификации тензоров Ниенхейса в точке. Тем не менее, приведенная классификация является достаточно тонкой: всем комплексным структурам соответствует одна точка $j_{\{0\} \infty} \in \mathscr{J}_{\infty}$.

\section{§4. Пространство линейных тензоров Ниенхейса}

Рассмотрим тензор Ниенхейса $N=N_{j}$ на фиксированном касательном пространстве $T_{x}$ как билинейное отображение $N: T_{x} \otimes T_{x} \rightarrow T_{x}$. Это отображение антилинейно и кососимметрично, $N \in \mathscr{N}_{j}$ (см. (10)). Никаких других ограничений нет.

ТЕОрема 5. Для любого тензора $N \in \mathscr{N}_{j_{0}}\left(T_{x}\right)$ существует почти комплексная структура $j$ на $\mathscr{O}(x)$ такая, что $j_{x}=j_{0} u N=\left(N_{j}\right)_{x}$. 
ДокАЗАТЕЛЬСтво. Пусть $\left(x^{i}\right)$ - система координат в окрестности точки $x$ такая, что векторы $\partial_{i}=\partial /\left.\partial x^{i}\right|_{x}$ образуют стандартньй комплексньй относительно $j_{0}$ базис в $T_{x}$. Тогда на $\mathscr{O}(x)$ имеем (считаем, что $x^{i}=0$ в точке $x$ )

$$
\begin{aligned}
j \partial_{2 r-1}=\partial_{2 r}+ & \sum a_{2 r}^{i} \partial_{i}, \quad j \partial_{2 r}=-\partial_{2 r-1}+\sum a_{2 r-1}^{i} \partial_{i}, \quad a_{s}^{i}(0)=0 ; \\
N_{j}\left(\partial_{2 s-1}, \partial_{2 t-1}\right)_{0}= & \sum\left(\frac{\partial a_{2 t}^{2 i-1}}{\partial x^{2 s}}(0)-\frac{\partial a_{2 s}^{2 i-1}}{\partial x^{2 t}}(0)+\frac{\partial a_{2 t}^{2 i}}{\partial x^{2 s-1}}(0)-\frac{\partial a_{2 s}^{2 i}}{\partial x^{2 t-1}}(0)\right) \partial_{2 i-1} \\
& +\sum\left(\frac{\partial a_{2 t}^{2 i}}{\partial x^{2 s}}(0)-\frac{\partial a_{2 s}^{2 i}}{\partial x^{2 t}}(0)-\frac{\partial a_{2 t}^{2 i-1}}{\partial x^{2 s-1}}(0)+\frac{\partial a_{2 s}^{2 i-1}}{\partial x^{2 t-1}}(0)\right) \partial_{2 i} \\
= & \sum\left(c_{s, t}^{2 i-1} \partial_{2 i-1}+c_{s, t}^{2 i} \partial_{2 i}\right) .
\end{aligned}
$$

Необходимо доказать, что при любом выборе констант $c_{s, t}^{i}, 1 \leqslant i \leqslant 2 n, 1 \leqslant s<t \leqslant n$, уравнение (26) имеет решение. Полагая $a_{2 t}^{i}\left(x^{1}, \ldots, x^{2 n}\right)=\sum_{s<t} c_{s, t}^{i} x^{2 s}$ и определяя $a_{2 t-1}^{i}$ из условия $j^{2}=-\mathbf{1}$, с учетом формулы (25) получаем требуемое.

ОПредЕЛЕнИЕ 3. Назовем пространством линейных тензоров Ниенхейса множество тензоров $\mathcal{N}_{j}^{(n)}$ на фиксированном комплексном линейном пространстве $V \simeq \mathbb{C}^{n}$, заданное формулой (10).

Рассмотрим отображение, сопоставляющее тензору Ниенхейса $N \in \mathscr{N}_{j}(V)$ на $V \simeq \mathbb{C}^{n}$ функцию $\mathfrak{n}_{N}: V \rightarrow 2 \mathbb{Z}_{+}$:

$$
\mathfrak{n}_{N}(\xi):=\operatorname{dim}\{\eta \mid N(\xi, \eta)=0\}=2 \operatorname{dim}_{\mathbb{C}}\{\eta \mid N(\xi, \eta)=0\} \in 2 \mathbb{Z}_{+} .
$$

Поскольку $\mathfrak{n}_{N}(\xi)=\mathfrak{n}_{N}(j \xi)$, функцию $\mathfrak{n}_{N}$ можно рассматривать как отображение $\mathfrak{n}_{N}$ : $P V \simeq \mathbb{C P}^{n-1} \rightarrow 2 \mathbb{Z}_{+}$. Эта функция полунепрерывна сверху по $\xi$ :

$$
\varlimsup_{\xi \rightarrow \xi_{0}} \mathfrak{n}_{N}(\xi) \leqslant \mathfrak{n}_{N}\left(\xi_{0}\right) \quad \forall \xi_{0} \in V
$$

она также полунепрерьвна сверху по $N$ :

$$
\varlimsup_{N^{\prime} \rightarrow N} \mathfrak{n}_{N^{\prime}}(\xi) \leqslant \mathfrak{n}_{N}(\xi) \quad \forall N \in \mathscr{N} .
$$

ОПРЕДЕЛЕНИЕ 4. Будем говорить, что линейный тензор Ниенхейса $N \in \mathscr{N}_{j}$ находится в общем положсении, если для почти всех $\xi$ выполнено равенство $\mathfrak{n}_{N}(\xi)=2$, т.е. ограничение $N$ на двумерные плоскости равно 0 только на комплексных прямых за исключением множества двумерных плоскостей меры 0.

Несложно показать, что условие в определении 4 является действительно условием общего положения: множество тензоров $N \in \mathscr{N}_{j}$ с $\mathfrak{n}_{N}(\xi)=2$ почти всюду является открытым в силу полунепрерывности, а всюду плотность легко доказывается с использованием следуюшего примера.

ПримеР 3. Рассмотрим билинейное кососимметричное отображение $A: \wedge^{2} \mathbb{R}^{n} \rightarrow \mathbb{R}^{n}$, определяемое формулой

$$
A(\xi, \eta)=\sum_{i=1}^{n}\left(\xi^{i} \eta^{i+1}-\xi^{i+1} \eta^{i}\right) e_{i}, \quad \xi=\sum_{i=1}^{n} \xi^{i} e_{i}, \quad \eta=\sum_{i=1}^{n} \eta^{i} e_{i}, \quad \xi^{n+1}=\xi^{1}, \quad \eta^{n+1}=\eta^{1} .
$$


Тензор $A$ удовлетворяет свойству: для почти всех векторов $\xi$ из равенства $A(\xi, \eta)=0$ следует параллельность векторов $\xi$ и $\eta$.

Определим комплексную структуру $j$ на $\mathbb{R}^{2 n}=\mathbb{R}_{1}^{n} \oplus \mathbb{R}_{2}^{n}$ формулой $j(\xi \oplus \eta)=(-\eta, \xi)$ и соответствующий антиинвариантньй тензор $N \in \mathcal{N}\left(\mathbb{R}^{2 n}\right), N: \wedge^{2} \mathbb{R}^{2 n} \rightarrow \mathbb{R}^{2 n}$, формулой

$$
N\left(\xi_{1} \oplus \eta_{1}, \xi_{2} \oplus \eta_{2}\right)=\left(A\left(\xi_{1}, \xi_{2}\right)-A\left(\eta_{1}, \eta_{2}\right)\right) \oplus\left(-A\left(\xi_{1}, \eta_{2}\right)-A\left(\eta_{1}, \xi_{2}\right)\right) .
$$

Легко видеть, что $\mathfrak{n}_{N}(\xi)=2$ для почти всех $\xi$.

Из определения 4 следует, что комплексные прямые можно выделить из двумерных плоскостей при помоши тензора Ниенхейса общего положения $N: \mathbb{C} \xi=\operatorname{Ker} N(\xi, \cdot)$ для почти всех $\xi$, а для других $\xi$ можно определить по непрерывности. Это позволяет ввести комплексную независимость векторов через тензор Ниенхейса $N$ общего положения: векторы $\xi_{1}, \ldots, \xi_{r}$ комплексно независимы, если для любого вектора $\xi$ из линейной оболочки $\Lambda=\left\langle\xi_{1}, \ldots, \xi_{r}\right\rangle$ общего положения и любого числа $s$ равенство $N\left(\xi_{s}, \xi\right)=0$ влечет $\xi=$ const $\cdot \xi_{s}$. Более того, по любому комплексно независимому набору из $n=\operatorname{dim}_{\mathbb{C}} V$ векторов общего положения в $V$ с помощью тензора $N$ канонически строится разложение на комплексные прямые $V=\bigoplus_{i=1}^{n} \mathbb{C}$, каждому вектору сопоставляется его двумерньй $N$-аннулятор.

ПРЕДЛОЖЕНИЕ 2. Пусть $N \in \wedge^{2} V^{*} \otimes V-$ кососимметричный тензор, антилинейный относительно двух комплексных структур $j_{1}$ и $j_{2}$ на линейном пространстве $V, N \in \mathscr{N}_{j_{1}} \cap \mathcal{N}_{j_{2}}$. Если $N$ - тензор общего полохсения, то $j_{1}= \pm j_{2}$.

ДокАЗАтЕльСтво. Рассмотрим произвольную комплексную прямую $\mathbb{C} \subset V$, т.е. двумерное подпространство, ограничение тензора $N$ на которое равно 0 в предположении, что $\mathbb{C}$ комплексно порождается вектором $\xi$ таким, что $\mathfrak{n}_{N}(\xi)=2$. Инвариантность этого подпространства относительно $j_{1}$ и $j_{2}$ очевидна. На этой прямой существует вектор $\xi \neq 0$ такой, что векторы $j_{1} \xi$ и $j_{2} \xi$ параллельны. Действительно, с точностью до гомотетии оператор комплексного умножения $j_{k}$ на евклидовой плоскости $\mathbb{R}^{2} \simeq \mathbb{C}$ совпадает с поворотом на некоторый угол:

$$
\varphi \mapsto \varphi+\delta_{k}(\varphi) \mapsto \varphi+\pi, \quad \varphi \in \mathbb{S}^{1}=\mathbb{R}^{1}(\bmod 2 \pi)
$$

Заменяя, если необходимо, $j_{2}$ на $-j_{2}$, можно считать, что $\delta_{1}(\varphi), \delta_{2}(\varphi) \in(0, \pi)$. Ни одно из неравенств $\delta_{1}(\varphi)<\delta_{2}(\varphi), \delta_{1}(\varphi)>\delta_{2}(\varphi)$ не может вьполняться для всех $\varphi$, поскольку $j_{1}^{2}=j_{2}^{2}=-\mathbf{1}$. Поэтому существует $\varphi$ такое, что $\delta_{1}(\varphi)=\delta_{2}(\varphi)$, т.е. существует вектор $\xi \neq 0$, для которого $j_{1} \xi=\alpha j_{2} \xi, \alpha \in \mathbb{R} \backslash\{0\}$.

Для любого вектора $\eta \notin \mathbb{C}=\mathbb{C} \xi$ имеем

$$
N\left(\xi, j_{1} \eta\right)=N\left(j_{1} \xi, \eta\right)=N\left(\alpha j_{2} \xi, \eta\right)=N\left(\xi, \alpha j_{2} \eta\right),
$$

т.е. $N\left(\xi, j_{1} \eta-\alpha j_{2} \eta\right)=0$. Но $j_{1} \eta, j_{2} \eta \in \mathbb{C} \eta$ (как объяснено вьше, $\mathbb{C} \eta$ определяется только тензором Ниенхейса $N)$, в то же время $\mathbb{C} \xi \cap \mathbb{C} \eta=\{0\}$. В силу равенства $\mathfrak{n}_{N}(\xi)=2$ имеем $j_{1} \eta=\alpha j_{2} \eta$ для любого вектора $\eta \notin \mathbb{C} \xi$, следовательно, и для любого $\eta \in V$ (по непрерьвности). Итак, $j_{1}=\alpha j_{2}$, откуда $\alpha= \pm 1$.

Рассмотрим линейное пространство $V$, две комплексных структуры $j_{1}$ и $j_{2}$ на нем и произвольный тензор Ниенхейса $N \in \mathscr{N}_{j_{1}} \cap \mathcal{N}_{j_{2}}$, необязательно общего вида. Предположим, что $N \not \equiv 0$. Тогда сушествует вектор $\theta=N(\xi, \eta) \neq 0$. Заметим, что $j_{1} j_{2} \theta=$ $N\left(j_{1} \xi, j_{2} \eta\right)=j_{2} j_{1} \theta$. Поэтому подпространство $\Theta=\left\langle\theta, j_{1} \theta, j_{2} \theta, j_{1} j_{2} \theta\right\rangle$ является $j_{1}-j_{2}$-инвариантньм. Заметим, что $\Theta$ распадается на сумму инвариантных подпространств: 
$\Theta=\left\langle\theta^{+}, j_{1} \theta^{+}\right\rangle \oplus\left\langle\theta^{-}, j_{1} \theta^{-}\right\rangle$, где $\theta^{+}=j_{1} \theta+j_{2} \theta$ и $\theta^{-}=j_{1} \theta-j_{2} \theta$ удовлетворяют соотношениям $j_{1} \theta^{+}=j_{2} \theta^{+}, j_{1} \theta^{-}=-j_{2} \theta^{-}$. Таким образом, если подпространство $\Theta$ четырехмерно, то существуют ненулевые векторы $\theta^{ \pm}$такие, что $j_{1} \theta^{ \pm}= \pm j_{2} \theta^{ \pm}$; если же $\Theta$ двумерно, то существует лишь один из таких векторов. Обозначим $\Pi^{+}=\left\{\xi \mid j_{1} \xi=j_{2} \xi\right\}$, $\Pi^{-}=\left\{\xi \mid j_{1} \xi=-j_{2} \xi\right\}, \Pi=\Pi^{+} \oplus \Pi^{-}$. Мы показали, что любой вектор $\theta \in \operatorname{Im} N(\cdot, \cdot)$ представим в виде суммы векторов из $\Pi^{+}$и $\Pi^{-}$. Заметим, что любой вектор $\theta \in V$ представим в виде $\theta=\theta_{(+)}+\theta_{(-)}$, где $\theta_{( \pm)}=\left(\theta \mp j_{1} j_{2} \theta\right) / 2$, причем $N\left(\theta_{(+)}, \Pi^{-}\right)=0$, $N\left(\theta_{(-)}, \Pi^{+}\right)=0$. Положим $K^{+}=\left\langle\xi \mid N\left(\xi, \Pi^{-}\right)=0\right\rangle, K^{-}=\left\langle\xi \mid N\left(\xi, \Pi^{+}\right)=0\right\rangle$. Мы доказали

ПРЕДЛОЖЕНИЕ 3. ДЛя подпространства $\widehat{\Pi} \subset V$, порожденного $\operatorname{Im} N(\cdot, \cdot)$, имеет место включение $\widehat{\Pi} \subset$ П. Кроме того, $\Pi^{+} \subset K^{+}, \Pi^{-} \subset K^{-}, V=K^{+}+K^{-}$. Пересечение $K^{+} \cap K^{-}$совпадает с пространством векторов $K=\operatorname{Ker} N(\cdot, \Pi):=$ $\{\xi \mid N(\xi, \Pi)=0\}$.

ПРИмеР 4. Рассмотрим линейное пространство $V^{8}=\mathbb{C}_{1} \oplus \mathbb{C}_{2} \oplus \mathbb{C}_{3} \oplus \mathbb{C}_{4}, \mathbb{C}_{s}=$ $\left\langle\partial_{2 s-1}, \partial_{2 s}\right\rangle, s=1,2,3,4$, со стандартной комплексной структурой $j_{1}$ и структурой $j_{2}$, отличающейся от $j_{1}$ только на базисных векторах $\mathbb{C}_{3}$, где $j_{2} \partial_{5}=\partial_{6}+\partial_{1}, j_{2} \partial_{6}=-\partial_{5}-\partial_{2}$. Рассмотрим тензор $N \in \mathscr{N}_{j_{1}} \cap \mathscr{N}_{j_{2}}$, заданньй условиями

$$
N\left(\partial_{1}, \partial_{3}\right)=\partial_{1}, \quad N\left(\partial_{5}, \partial_{7}\right)=\partial_{1}, \quad N\left(\mathbb{C}_{1} \oplus \mathbb{C}_{2}, \mathbb{C}_{3} \oplus \mathbb{C}_{4}\right)=0 .
$$

Имеем $\Pi^{-}=0, \Pi=\Pi^{+}=\mathbb{C}_{1} \oplus \mathbb{C}_{2} \oplus \mathbb{C}_{4}, K^{-}=\operatorname{Ker} N(\cdot, \Pi)=\mathbb{C}_{4}, K^{+}=V^{8}$, $\operatorname{Ker} N(\cdot, \cdot)=0$.

\section{§5. Почти комплексные структуры общего вида и их классификация}

В этом параграфе мы изучим обшие свойства тензоров Ниенхейса и упростим следствие из теоремы 3 для почти комплексных структур общего вида.

ОПРЕДЕЛЕниЕ 5. Будем говорить, что почти комплексная структура $j$ на $\mathscr{O}(x) u м e-$ em общий вид в точке $x$, если линейный тензор Ниенхейса $\left(N_{j}\right)_{x}$ в $T_{x}$ обладает свойством общего положения $\mathfrak{n}_{N_{j}}(\xi)=2$ для почти всех $\xi$. Кроме того, будем считать, что пара $(\mathscr{O}(x), j)$ неизоморфна $(\mathscr{O}(x),-j)$.

Отметим, что множество точек, где почти комплексная структура имеет общий вид, является открытым.

ЗАмЕчаниЕ 4. Почти комплексная структура $j$ на $\mathscr{O}(x)$ общего вида неизоморфна структуре $-j$. Действительно, для инварианта, введенного в $\S 2$, имеем $N_{-j}^{(2)}=-N_{j}^{(2)}$, и для почти комплексной структуры $j$ общего вида тензоры $N_{j}^{(2)}$ и $-N_{j}^{(2)}$ (на фиксированном касательном пространстве) неизоморфны. Заметим, что $\left(T_{x}, j_{x}\right) \simeq\left(T_{x},-j_{x}\right)$ и $N_{-j}=N_{j}$, откуда следует, что $j \simeq-j\left(\bmod \mu_{x}^{2}\right)$ на $\mathscr{O}(x)$, но почти всегда $j \not z-j\left(\bmod \mu_{x}^{3}\right)$, и тензор $N_{j}^{(2)}$ является первым образующим инвариантом в $\mathscr{A}_{j}^{\infty}$, различающим в общем случае почти комплексные структуры $j$ и $-j$.

ПримеР 5. Рассмотрим почти комплексную структуру в $\mathbb{R}^{4}$, определенную равенствами

$$
\begin{array}{ll}
j \partial_{1}=\partial_{2}, & j \partial_{3}=\partial_{4}+\left(x^{2}\right)^{2} \partial_{1}+\varepsilon\left(x^{3}\right)^{2} \partial_{2}, \\
j \partial_{2}=-\partial_{1}, & j \partial_{4}=-\partial_{3}-\left(x^{2}\right)^{2} \partial_{2}+\varepsilon\left(x^{3}\right)^{2} \partial_{1} .
\end{array}
$$

При $\varepsilon=0$ почти комплексные структуры $j$ и $-j$ изоморфны, а при $\varepsilon \neq 0$ неизоморфны. Легко видеть, что при $\varepsilon \neq 0$ приведенная почти комплексная структура имеет общий вид. 
ТЕОРема 6. Предположим, что почти комплексные структуры $j_{L}$ на $\mathscr{O}_{L}(x)$ и $j_{M}$ на $\mathscr{O}_{M}(y)$ имеют общий вид.

1. Пусть существует отобрахсение $u: \mathscr{O}_{L}(x) \rightarrow \mathscr{O}_{M}(y)$, сопрягающее $N_{j_{L}} c N_{j_{M}}$ и являющееся диффеоморфизмом на образ. Тогда и является либо псевдоголоморфным, либо антипсевдоголоморфным отображением: $j_{M} \circ u_{*}= \pm u_{*} \circ j_{L}$.

2. Если тензоры Ниенхейса сопря⿻ень формально, $N_{j_{M}} \circ u_{*}^{\wedge 2} \equiv u_{*} \circ N_{j_{L}}\left(\bmod \mu_{x, y}^{\infty}\right)$, и размерности многообразий $L$ и $M$ совпадают, то почти комплексная структура $j_{L}$ формально әквивалентна в точности одной из структур $j_{M}$ или- $j_{M}$.

ДокАЗАтЕльство. Рассмотрим случай гладкой сопряженности тензоров Ниенхейса (формальный получается переходом к рядам). Пусть $N_{j_{M}} \circ \wedge^{2} u_{*}=u_{*} \circ N_{j_{L}}$. Выберем комплексные параллелизации $T_{x^{\prime}} L=\bigoplus_{1}^{l} \mathbb{C}_{\text {и }} T_{y^{\prime}} M=\bigoplus_{1}^{m} \mathbb{C}$ такие, что отображение $u_{*}$ вкладывает одну в другую. Образ $u(L)$ является $j_{M}$-инвариантньм. Поэтому мы можем рассматривать случаи многообразий одинаковой размерности $2 l=2 m$ и предполагать, что $u$ - диффеоморфизм. Мы имеем в точке $y^{\prime} \in u(L) \subset M$ тензор Ниенхейса $N_{j_{M}}=u_{*} \circ N_{j_{L}} \circ\left(u_{*}^{\wedge 2}\right)^{-1}$ и две комплексные структуры $j_{M}$ и $u_{*} \circ j_{L} \circ u_{*}^{-1}$ на $T_{y^{\prime}}$, относительно которых $N_{j_{M}}$ антилинеен. В силу предложения $2 j_{M} u_{*}= \pm u_{*} j_{L}$ и так как почти комплексная структура $j$ общего вида неизоморфна $-j$, теорема доказана.

ЗАмечаниЕ 5. Теорема неверна для почти комплексных структур не общего положения. Если $j_{L}$ на $\mathscr{O}_{L}(x)$ и $j_{M}$ на $\mathscr{O}_{M}(y)$ - структуры общего положения, то тензоры Ниенхейса почти комплексных структур $j_{L} \oplus j_{M}$ и $j_{L} \oplus\left(-j_{M}\right)$ на $\mathscr{O}_{L}(x) \times \mathscr{O}_{M}(y)$ совпадают, хотя сами структуры ни изоморфны и ни антиизоморфны.

\section{§6. Два частных случая}

6.1. Почти комплексные структуры в $\mathbb{R}^{4}$. Рассмотрим почти комплексную структуру $j$ в $\mathbb{R}^{4}$ в окрестности нуля. Предположим, что $\left(N_{j}\right)_{0} \neq 0$. В этом случае образ $\operatorname{Im} N_{j}(\cdot, \cdot)$ двумерен и комплексно порожден вектором $N_{j}(\xi, \eta) \neq 0$ для любых комплексно независимых векторов $\xi$ и $\eta$. Рассмотрим двумерное распределение $\Pi_{x}^{2}=\operatorname{Im}\left(N_{j}\right)_{x} \subset T_{x}=T_{x} \mathbb{R}^{4}$. Оно является инвариантом почти комплексной структуры, поэтому инварианты этого распределения приводят к инвариантам структуры (заметим, что любое регулярное 2-распределение на четырехмерном пространстве может быть реализовано как образ тензора Ниенхейса некоторой почти комплексной структуры). Важным инвариантом распределения является инвариант Танаки (см. [13], [14]) набор градуированных алгебр Ли $\mathfrak{m}(x)$ для каждой точки $x$, присоединенных к фильтрованным алгебрам $\left\{D^{p}(x) \subset T_{x}\right\}_{p \geqslant 1}$, где модуль сечений $\mathscr{D}(p)$ распределения $D^{p}$ определяется как $(p-1)$-я производная: $\mathscr{D}^{(p)}=\partial^{(p-1)} \mathscr{D}^{(1)}=\partial^{(p-2)} \mathscr{D}^{(1)}+\left[\mathscr{D}^{(1)}, \partial^{(p-2)} \mathscr{D}^{(1)}\right]$; здесь $[\cdot, \cdot]$ - коммутатор векторных полей, а $\partial^{(0)} \mathscr{D}^{(1)}=\mathscr{D}^{(1)}$ - сечения первоначального распределения $D^{1}$; умножение Ли индуцировано коммутатором векторных полей.

Предположим, что первая производная распределения $\Pi_{*}^{2}$ нетривиальна в точке 0 , т.е. $\left(\partial^{(1)} \Pi^{2}\right)_{0} \neq \Pi_{0}^{2}$. Тогда мы имеем трехмерное распределение $\Pi^{3}=\partial^{(1)} \Pi^{2}$ в $\mathscr{O}(0)$. В пространстве $\Pi_{x}^{3} \subset T_{x}$ существуют векторы $\xi_{i}=\xi_{i}(x) \neq 0, i=1,2,3$, такие, что $N_{j}\left(\xi_{1}, \xi_{3}\right)=\xi_{1}, N_{j}\left(\xi_{2}, \xi_{3}\right)=-\xi_{2}$. Обозначим одномерные подпространства, порожденные векторами $\xi_{1}$ и $\xi_{2}$, через $\Upsilon_{1}=\left\langle\xi_{1}\right\rangle$ и $\Upsilon_{2}=\left\langle\xi_{2}\right\rangle$ соответственно, $\Pi_{x}^{2}=\left(\Upsilon_{1}\right)_{x} \oplus\left(\Upsilon_{2}\right)_{x}$. Заметим, что если подпространство $\left(\Pi_{x}^{3}\right)^{+} \subset \Pi_{x}^{3} \backslash \Pi_{x}^{2}$, в котором лежит вектор $\xi_{3}$, фиксировано, то этот вектор определен с точностью до $\Pi_{x}^{2}$-сдвигов: $\xi_{3} \mapsto \xi_{3}+\alpha_{1} \xi_{1}+\alpha_{2} \xi_{2}$. При замене полупространства $\xi_{3} \mapsto \widetilde{\xi}_{3}=-\xi_{3}$ векторы $\xi_{1}$ и $\xi_{2}$ (следовательно, и распределения $\Upsilon_{1}$ и $\Upsilon_{2}$ ) поменяются местами: $\widetilde{\xi}_{1}= \pm \xi_{2}, \widetilde{\xi}_{2}= \pm \xi_{1}$. Выбор полупространства 
в $\Pi_{x}^{3} \backslash \Pi_{x}^{2}$, т.е. ориентации в $\Theta_{x}^{1}=\Pi_{x}^{3} / \Pi_{x}^{2}$, определяет порядок следования распределений $\Upsilon_{1}$ и $\Upsilon_{2}$; изменение этой ориентации меняет нумерацию. Другими словами, в двумерном пространстве $\Theta_{x}^{1} \times P\left(\Pi_{x}^{2}\right)$ имеется каноническая ориентация. Назовем ее $\Theta$-ориентаиией. Заметим также, что в пространстве $\Pi_{x}^{3}$ выделена пара двумерных аффинных подпространств: $\left\{ \pm \xi_{3}+\alpha_{1} \xi_{1}+\alpha_{2} \xi_{2}\right\}$, т.е. на $\Theta_{x}^{1}$ фиксирована метрика. Назовем ее $\Theta$-метрикой.

Рассмотрим теперь для подпространства $\Pi_{x}^{3} \subset T_{x}$ фактор $\Xi_{x}^{1}=T_{x}^{4} / \Pi_{x}^{3}$. Существует вектор $\xi_{4} \notin \Pi_{x}^{3}$ такой, что $N_{j}\left(\xi_{1}, \xi_{4}\right)=\xi_{2}, N_{j}\left(\xi_{2}, \xi_{4}\right)=-\xi_{1}$. В своем полупространстве $T_{x}^{4} \backslash \Pi_{x}^{3}$ он определен с точностью до $\Pi_{x}^{2}$-сдвигов: $\xi_{4} \mapsto \xi_{4}+\alpha_{1} \xi_{1}+\alpha_{2} \xi_{2}$, поэтому на $\Xi_{x}^{1}$ имеется естественная метрика. Назовем ее $\Xi$-метрикой. При замене полупространства $\xi_{4} \mapsto \widetilde{\xi}_{4}=-\xi_{4}$ в одном из подпространств $\Upsilon_{1}$ или $\Upsilon_{2}$ меняется ориентация: $\xi_{1} \mapsto-\xi_{1}$ или $\xi_{2} \mapsto-\xi_{2}$. Поэтому определена естественная ориентация пространства $\Upsilon_{1}^{1} \times \Upsilon_{2}^{1} \times \Xi^{1}$. Назовем ее $\Xi$-ориентаиией.

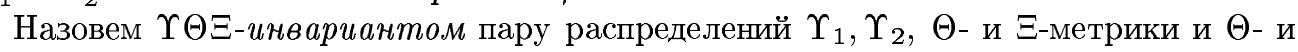
$\Xi$-ориентации. Из рассуждений, приведенных выше, и теоремы 6 следует

ТЕОРема 7. Пусть для почти комплексных структур $j_{1}$ на $\mathscr{O}_{\mathbb{R}^{4}}(x)$ и $j_{2}$ на $\mathscr{O}_{\mathbb{R}^{4}}(y)$ тензоры Ниенхейса не равны 0 и первая производная распределения $\Pi^{2}$ нетривиальна, $\partial^{(1)} \Pi^{2} \neq \Pi^{2}$. Псевдоголоморфное или антипсевдоголоморфное отображение $и:\left(\mathscr{O}_{\mathbb{R}^{4}}(x), j_{1}\right) \rightarrow\left(\mathscr{O}_{\mathbb{R}^{4}}(y), j_{2}\right)$ существует тогда и только тогда, когда существует отображсение $\mathscr{O}_{\mathbb{R}^{4}}(x) \rightarrow \mathscr{O}_{\mathbb{R}^{4}}(y)$, переводящее один $\Upsilon \Theta \Xi$-инвариант в другой.

ЗАмЕчАниЕ 6 . В случае, если вторая производная распределения $\Pi^{2}$ не совпадает с первой, $\partial^{(2)} \Pi^{2} \neq \partial^{(1)} \Pi^{2} \neq \Pi^{2}$, т.е. $\left(\partial^{(2)} \Pi^{2}\right)_{x}=T_{x}^{4}$, инвариант Танаки имеет подлежащее пространство вида $\mathfrak{m}(x) \simeq \Pi_{x}^{2} \oplus \Theta_{x}^{1} \oplus \Xi_{x}^{1}$. При этом в силу градуированности умножение Ли задается 2-формой на $\Pi_{x}^{2}$ со значениями в $\Theta_{x}^{1}$ и 1-формой на $\Pi_{x}^{2}$ со значениями в $\operatorname{Hom}\left(\Theta_{x}^{1}, \Xi_{x}^{1}\right)$. В силу существования $\Theta$ - и $\Xi$-метрик, $\Theta$ - и $\Xi$-ориентаций и разложения $\Pi_{x}^{2}=\left(\Upsilon_{1}\right)_{x} \oplus\left(\Upsilon_{2}\right)_{x}$ структура алгебры Ли на $\mathfrak{m}(x)$ задается элементами $\omega_{x}^{2} \in\left(\Upsilon_{1}\right)_{x}^{*} \otimes\left(\Upsilon_{2}\right)_{x}^{*}=\operatorname{Hom}\left(\Upsilon_{1}, \Upsilon_{2}^{*}\right)_{x}$ и $\omega_{x}^{1} \in\left(\Upsilon_{1}\right)_{x}^{*} \oplus\left(\Upsilon_{2}\right)_{x}^{*}$. Таким образом, инвариант Танаки порождает еще два инварианта: $\omega^{1}$ и $\omega^{2}$.

6.2. Лиевы почти комплексные структуры. Рассмотрим почти комплексную структуру $j$ и ее тензор Ниенхейса $N_{j}$. Он определяет билинейное кососимметричное отображение $N_{j}: \mathscr{D} \otimes \mathscr{D} \rightarrow \mathscr{D}$ на модуле $\mathscr{D}$ векторных полей. В общем случае, это отображение не является умножением Ли. Действительно, из тождества Якоби

$$
N(\xi, N(\eta, j \zeta))+N(\eta, N(j \zeta, \xi))+N(j \zeta, N(\xi, \eta))=0
$$

для элементов $\xi, \eta, j \zeta$ следует

$$
j N(\xi, N(\eta, \zeta))+j N(\eta, N(\zeta, \xi))-j N(\zeta, N(\xi, \eta))=0
$$

что вместе с тождеством Якоби для $\xi, \eta, \zeta$ дает

$$
N(\xi, N(\eta, \zeta)) \equiv 0
$$

Итак, $(\mathscr{D}, N(\cdot, \cdot))$ является алгеброй Ли тогда и только тогда, когда выполняется (27). Обозначим через $\Pi_{x} \subset T_{x}$ подпространство, порожденное $\operatorname{Im} N(\cdot, \cdot)$. Положим $A_{x}=\{\xi \mid N(\xi, \cdot) \equiv 0\}$. Формула $(27)$ равносильна включению $(j$-инвариантных) подрасслоений $\Pi \subset A \subset T X$. 
ОПРЕДЕЛЕНИЕ 6. Назовем почти комплексную структуру $j$ на $X$ лиевой, если ее тензор Ниенхейса $N_{j}$ определяет структуру алгебры Ли на модуле $\mathscr{D}$. Назовем лиеву почти комплексную структуру невырожденной в точке $x$, если $\Pi_{x}=A_{x}$ и для почти всякого вектора $\xi \in T_{x}$ из $N_{j}(\xi, \eta)=0$ следует, что $\eta \in \mathbb{C} \xi \oplus \Pi_{x}$.

Из теоремы 6 следует, что тензор Ниенхейса невырожденной лиевой почти комплексной структуры определяет почти комплексную структуру на трансверсали к П с точностью до знака и прибавления элемента из $A$. Отсюда в силу $\Pi=A$ следует определенность с точностью до знака почти комплексной структуры на $A$. Итак, если две невырожденные лиевы почти комплексные структуры имеют одинаковые тензоры Ниенхейса, то они могут отличаться только на сечение $\sigma$ расслоения $\bigcup_{x} \operatorname{Hom}\left(T_{x} / \Pi_{x}, \Pi_{x}\right)$, которое мы назовем связывающим сечением.

ТЕОРема 8. Лиева почти комплексная структура $ј$ задает разрешимую алгебру Ли $\mathscr{G}=\left(\mathscr{D}, N_{j}(\cdot, \cdot)\right)$ с рангом разрешимости 2: $\mathscr{G}^{(2)}=0$. Две невырохденные лиевы почти комплексные структуры эквивалентны или антиэквивалентны (формально или гладко), если әквивалентны соответствующие алгебры Ли $(\mathscr{D}, N(\cdot, \cdot))$ и связывающее сечение $\sigma \in \operatorname{Hom}\left(\mathscr{G} / \mathscr{G}^{(1)}, \mathscr{G}^{(1)}\right)$ равно 0.

Таким образом, теорема приводит к исследованию деформаций умножений Ли специального вида; заметим, что не все такие деформации встречаются (см. замечание 3 ).

ЗАмечание 7. Рассмотрим ( под) аләебру Ниенхейса $\mathscr{A}_{j}^{\mathcal{N}}$, порожденную комплексной структурой $j$ при помощи алгебраического коммутатора

$$
[A, B]=B \bar{\wedge} A-(-1)^{\operatorname{deg} A \operatorname{deg} B} A \bar{\wedge} B
$$

и скобки Ниенхейса $\left[[\cdot, \cdot]\right.$, в алгебре векторнозначных форм $\Omega^{*} \otimes \mathscr{D}$ на многообразии $X$ (см. [12], [6], обозначения схожи с [8]). Здесь $\Omega^{*}$ - алгебра скалярнозначных дифференциальных форм, а $\mathscr{D}$ - модуль векторных полей. Заметим, что в случае лиевой почти комплексной структуры $\mathscr{A}_{j}^{\mathcal{N}}=\left\langle j, N_{j}, j \circ N_{j}\right\rangle$. Действительно, как показьвают соотношения (2.9), (2.10), (2.16), (5.6), (5.8), (5.22), (5.23) в [12], с учетом тождества Якоби $N_{j} \bar{\wedge} N_{j}=0$ для $N_{j}$ имеют место следующие равенства:

$$
\begin{gathered}
{[j, j]=0, \quad\left[j, N_{j}\right]=-3 j \wedge N_{j}=\frac{3}{2} N_{j} \bar{\wedge} j=-3 j \circ N_{j}, \quad\left[j, j \circ N_{j}\right]=3 N_{j},} \\
{\left[N_{j}, N_{j}\right]=2 N_{j} \bar{\wedge} N_{j}=0, \quad\left[N_{j}, j \circ N_{j}\right]=0, \quad\left[j \circ N_{j}, j \circ N_{j}\right]=0,} \\
\left.\llbracket j, j]=N_{j}, \quad \llbracket j, N_{j} \rrbracket=0, \quad \| j, j \circ N_{j}\right]=-\frac{1}{2} N_{j} \bar{\wedge} N_{j}=0, \\
\llbracket N_{j}, N_{j} \rrbracket=0, \quad \llbracket N_{j}, j \circ N_{j} \rrbracket=0, \quad \llbracket j \circ N_{j}, j \circ N_{j} \|=0 .
\end{gathered}
$$

Следующий пример показьвает, что значения $\left(\mathscr{A}_{j}^{\mathcal{N}}\right)_{x}$ в точке $x$ недостаточно для формальной классификации почти комплексной структуры, т.е. $\mathscr{A}_{j}^{\mathcal{N}} \neq \mathscr{A}_{j}^{\infty}$ (см. начало 2$)$.

ПРИмеР 6. Рассмотрим невырожденную лиеву почти комплексную структуру, определенную формулами

$$
\begin{array}{lll}
j \partial_{1}=\partial_{2}, & j \partial_{3}=\partial_{4}+f\left(x^{5}\right) \partial_{1}, & j \partial_{5}=\partial_{6} \\
j \partial_{2}=-\partial_{1}, & j \partial_{4}=-\partial_{3}-f\left(x^{5}\right) \partial_{2}, & j \partial_{6}=-\partial_{5}
\end{array}
$$


Тензор Ниенхейса на базисных векторах определяется равенствами

$$
\begin{gathered}
N_{j}\left(\partial_{1}, \cdot\right)=N_{j}\left(\partial_{2}, \cdot\right)=N_{j}\left(\partial_{3}, \partial_{4}\right)=N_{j}\left(\partial_{5}, \partial_{6}\right)=0 \\
N_{j}\left(\partial_{3}, \partial_{5}\right)=-N_{j}\left(\partial_{4}, \partial_{6}\right)=f^{\prime}\left(x^{5}\right) \partial_{2}, \quad N_{j}\left(\partial_{3}, \partial_{6}\right)=N_{j}\left(\partial_{4}, \partial_{5}\right)=f^{\prime}\left(x^{5}\right) \partial_{1} .
\end{gathered}
$$

Видно, что структура $j$ формально не классифищируется алгеброй $\left(\mathscr{A}_{j}^{\mathcal{N}}\right)_{x}$. Действительно, при $f\left(x^{5}\right)=\varepsilon\left(x^{5}\right)^{2}$ имеется инвариант $\left(d N_{j}\right)_{0}$, а при $f\left(x^{5}\right)=x^{5}+\varepsilon\left(x^{5}\right)^{2}$ алгебры $\mathscr{A}_{j}^{\mathcal{N}}$ тоже недостаточно, чтобы различить структурыпри различных $\varepsilon$, хотя тензоры Ниенхейса во всех точках из окрестности $x$ сопряжены.

ЗАмЕчаниЕ 8. Рассмотрим тензор Ниенхейса лиевой почти комплексной структуры $j$ на $\mathscr{O}(x)$. Существует возрастающая $j$-инвариантная фильтрация $\Theta_{k} \subset T_{x}$, где $\Theta_{k}$ порождено $\operatorname{Im} d^{k} N(\cdot, \cdot, *)$. Точке $x$ можно сопоставить следующий инвариант: присоединенную градуированную $j$-комплексную алгебру Ли относительно $N$-произведения $\oplus V_{k}, V_{k}=\Theta_{k} / \Theta_{k-1}$.

Автор благодарит профессора В. В. Льчагина за внимание к работе и полезные обсуждения.

\section{СПИСОК ЦИТИРОВАННОЙ ЛИТЕРАТУРЫ}

[1] Nijenhuis A., Woolf W.B. Some integration problems in almost-complex and complex manifolds // Ann. of Math. (2). 1963. V. 77. P. 424-489.

[2] Gromov M. Pseudo holomorphic curves in symplectic manifolds // Invent. Math. 1985. V. 82. P. 307-347.

[3] McDuff D., Salamon D. J-Holomorphic Curves and Quantum Cohomology. Univ. Lecture Ser. V. 6. Providence (R.I.): Amer. Math. Soc., 1994.

[4] Ньюландер А., Ниренберг Л. Комплексные аналитические координаты на почти комплексных многообразиях // Математика. 1959. Т. 3. №6. С. 131-144.

[5] Kuranishi M. New proof for the existence of locally complete families of complex structures // Proc. Conf. on Complex Analysis (Minneapolis, 1964). Berlin: Springer, 1965. P. 142-154.

[6] Алексеев Д. В., Виноградов А. М., Лычагин В. В. Основные идеи и понятия дифференциальной геометрии. Итоги науки и техн. Соврем. пробл. матем. Фундамент. направления. Т. 28. М.: ВИНИТИ, 1988.

[7] Виноградов А. М., Красильщик И. С., Лычагин В. В. Введение в геометрию нелинейных дифференциальных уравнений. М.: Наука, 1986.

[8] Kumpera A., Spencer D. Lie Equations. V. 1: General Theory. Ann. of Math. Stud. V. 73. Princeton (N.J.): Princeton Univ. Press, Univ. of Tokyo Press, 1972.

[9] Пале Р. Семинар по теореме Атьи-Зингера об индексе. М.: Мир, 1970.

[10] Gauduchon P. The canonical almost complex structure on the manifold of 1-jets of pseudo-holomorphic mappings between two almost complex manifolds. Appendix to Ch. II // Holomorphic Curves in Symplectic Geometry / ed. M. Audin, J. Lafontaine. Progr. Math. V. 117. Boston (M.A.): Birkhäuser, 1994. P. 69-73.

[11] Лихнерович А. Теория связностей в целом и группы голономий. М.: ИЛ, 1960.

[12] Frölicher A., Nijenhuis A. Theory of vector-valued differential forms. I: Derivations in the graded ring of differential forms // Proc. Konink. Nederl. Akad. Wetensch. Ser. A. 1956. V. 59. P. 338-359.

[13] Tanaka N. On differential systems, graded Lie algebras and pseudo-groups // J. Math. Kyoto Univ. 1970. V. 10. P. 1-82.

[14] Yamaguchi K. Differential systems associated with simple graded Lie algebras // Progress in Differential Geometry. Adv. Stud. Pure Math. V. 22. Tokyo: Kinokuniya, 1993. P. 413-494.

Московский государственный технический университет им. Н. Э. Баумана

Поступило

E-mail: borkru@difgeo.math.msu.su

18.09 .96

Исправленный вариант

10.11.97 